Алгебра и анализ

Том. 16 (2004), вып. 1
St. Petersburg Math. J. Vol. 16 (2005), No. 1, Pages 181-209 S 1061-0022(04)00847-7

Article electronically published on December 17, 2004

\title{
SPECTRAL SHIFT FUNCTION IN STRONG MAGNETIC FIELDS
}

\author{
V. BRUNEAU, A. PUSHNITSKI, AND G. RAIKOV \\ Dedicated to Professor Mikhail Birman on the occasion of his 75th birthday
}

\begin{abstract}
The three-dimensional Schrödinger operator $H$ with constant magnetic field of strength $b>0$ is considered under the assumption that the electric potential $V \in L^{1}\left(\mathbb{R}^{3}\right)$ admits certain power-like estimates at infinity. The asymptotic behavior as $b \rightarrow \infty$ of the spectral shift function $\xi\left(E ; H, H_{0}\right)$ is studied for the pair of operators $\left(H, H_{0}\right)$ at the energies $E=\mathcal{E} b+\lambda, \mathcal{E}>0$ and $\lambda \in \mathbb{R}$ being fixed. Two asymptotic regimes are distinguished. In the first regime, called asymptotics far from the Landau levels, we pick $\mathcal{E} / 2 \notin \mathbb{Z}_{+}$and $\lambda \in \mathbb{R}$; then the main term is always of order $\sqrt{b}$, and is independent of $\lambda$. In the second asymptotic regime, called asymptotics near a Landau level, we choose $\mathcal{E}=2 q_{0}, q_{0} \in \mathbb{Z}_{+}$, and $\lambda \neq 0$; in this case the leading term of the SSF could be of order $b$ or $\sqrt{b}$ for different $\lambda$.
\end{abstract}

\section{§1. INTRODUCTION}

The main object of investigation in the present paper is the spectral shift function (SSF) for the three-dimensional Schrödinger operator with constant magnetic field, perturbed by an electric potential that decays sufficiently fast at infinity. We recall the abstract setting in which the SSF for a pair of selfadjoint operators occurs. First, let $\mathcal{T}_{0}$ and $\mathcal{T}$ be two selfadjoint operators acting in the same Hilbert space, and let $\mathcal{T}-\mathcal{T}_{0} \in S_{1}$, where $S_{1}$ denotes the trace class. Then there exists a unique function $\xi\left(\cdot ; \mathcal{T}, \mathcal{T}_{0}\right) \in L^{1}(\mathbb{R})$ such that the Lifshits-Krein trace formula

$$
\operatorname{Tr}\left(f(\mathcal{T})-f\left(\mathcal{T}_{0}\right)\right)=\int_{\mathbb{R}} \xi\left(E ; \mathcal{T}, \mathcal{T}_{0}\right) f^{\prime}(E) d E
$$

is true for every $f \in C_{0}^{\infty}(\mathbb{R})$ (see [19] or, e.g., 31, Theorem 8.3.3]). Now, let $\mathcal{H}_{0}$ and $\mathcal{H}$ be two lower-bounded selfadjoint operators acting in the same Hilbert space. Assume that there exists $\gamma>0$ such that for some $\lambda_{0} \in \mathbb{R}$ lying strictly below the infima of the spectra of $\mathcal{H}_{0}$ and $\mathcal{H}$ we have

$$
\left(\mathcal{H}-\lambda_{0}\right)^{-\gamma}-\left(\mathcal{H}_{0}-\lambda_{0}\right)^{-\gamma} \in S_{1}
$$

Set

$$
\xi\left(E ; \mathcal{H}, \mathcal{H}_{0}\right):= \begin{cases}-\xi\left(\left(E-\lambda_{0}\right)^{-\gamma} ;\left(\mathcal{H}-\lambda_{0}\right)^{-\gamma},\left(\mathcal{H}_{0}-\lambda_{0}\right)^{-\gamma}\right) & \text { if } E>\lambda_{0}, \\ 0 & \text { if } E \leq \lambda_{0} .\end{cases}
$$

Then we have

$$
\operatorname{Tr}\left(f(\mathcal{H})-f\left(\mathcal{H}_{0}\right)\right)=\int_{\mathbb{R}} \xi\left(E ; \mathcal{H}, \mathcal{H}_{0}\right) f^{\prime}(E) d E, \quad f \in C_{0}^{\infty}(\mathbb{R})
$$

2000 Mathematics Subject Classification. Primary 35J10.

Key words and phrases. Schrödinger operator, spectral shift function, asymptotics.

V. Bruneau and G. Raikov were supported by the Chilean Science Foundation Fondecyt (grants nos. 1020737 and 7020737). A. Pushnitski was supported by the EPSRC (grant no. GR/R53210/01). 
(see [31, Theorem 8.9.1]). The function $\xi\left(\cdot ; \mathcal{H}, \mathcal{H}_{0}\right)$ is called the SSF for the pair $\left(\mathcal{H}, \mathcal{H}_{0}\right)$ of operators; it does not depend on the particular choice of $\gamma$ and $\lambda_{0}$ in (1.2) and belongs to the class $L^{1}\left(\left(\lambda_{0}, \infty\right),\left(E-\lambda_{0}\right)^{-\gamma-1} d E\right)$.

If $E$ lies below the infimum of the spectrum of $\mathcal{H}_{0}$, then $\mathcal{H}$ can possess only finitely many eigenvalues below $E$, and

$$
\xi\left(E ; \mathcal{H}, \mathcal{H}_{0}\right)=-N(E ; \mathcal{H}),
$$

where $N(E ; \mathcal{H})$ denotes the number of the eigenvalues of $\mathcal{H}$ lying on the interval $(-\infty, E]$ and counted with the multiplicities. On the other hand, for almost every $E$ in the absolutely continuous spectrum of $\mathcal{H}_{0}$, the $\operatorname{SSF} \xi\left(E ; \mathcal{H}, \mathcal{H}_{0}\right)$ is related to the scattering determinant $\operatorname{det} S\left(E ; \mathcal{H}, \mathcal{H}_{0}\right)$ for the pair $\left(\mathcal{H}, \mathcal{H}_{0}\right)$ by the Birman-Kreün formula

$$
\operatorname{det} S\left(E ; \mathcal{H}, \mathcal{H}_{0}\right)=e^{-2 \pi i \xi\left(E ; \mathcal{H}, \mathcal{H}_{0}\right)}
$$

(see [9] or [31, 8.4]).

In the present paper the role of $\mathcal{H}_{0}$ is played by the operator $H_{0}:=(i \nabla+\mathbf{A})^{2}-b$, essentially selfadjoint on the Schwartz class $\mathcal{S}\left(\mathbb{R}^{3}\right)$. Here the magnetic potential $\mathbf{A}=$ $\left(-\frac{b x_{2}}{2}, \frac{b x_{1}}{2}, 0\right)$ generates the constant magnetic field $\mathbf{B}=\operatorname{curl} \mathbf{A}=(0,0, b), b>0$. It is well known that $\sigma\left(H_{0}\right)=\sigma_{\mathrm{ac}}\left(H_{0}\right)=[0, \infty)$ (see [3]), where $\sigma\left(H_{0}\right)$ denotes the spectrum of $H_{0}$, and $\sigma_{\mathrm{ac}}\left(H_{0}\right)$ denotes its absolutely continuous spectrum. Moreover, the Landau levels $2 b q, q \in \mathbb{Z}_{+}$, play the role of thresholds in the spectrum of $H_{0}$ (here and in what follows $\mathbb{Z}_{+}$denotes the set of all nonnegative integers).

For $\mathbf{x}=\left(x_{1}, x_{2}, x_{3}\right) \in \mathbb{R}^{3}$ we denote by $X_{\perp}=\left(x_{1}, x_{2}\right)$ the variables in the plane perpendicular to the magnetic field. We assume that $V$ satisfies

$$
V \not \equiv 0, \quad V \in C\left(\mathbb{R}^{3}\right), \quad|V(\mathbf{x})| \leq C_{0}\left\langle X_{\perp}\right\rangle^{-m_{\perp}}\left\langle x_{3}\right\rangle^{-m_{3}}, \quad \mathbf{x} \in \mathbb{R}^{3},
$$

with $C_{0}>0, m_{\perp}>2, m_{3}>1$, and $\langle x\rangle:=\left(1+|x|^{2}\right)^{1 / 2}, x \in \mathbb{R}^{d}, d \geq 1$. Set $H:=H_{0}+V$. Obviously, $\inf \sigma(H) \geq-C_{0}$. In this paper, the role of the perturbed operator $\mathcal{H}$ is played by $H$. By (1.5) and the diamagnetic inequality (see, e.g., [3]), the operator $V^{1 / 2}\left(H_{0}-\lambda_{0}\right)^{-1}$ with $\lambda_{0}<0$ is Hilbert-Schmidt. Therefore, the resolvent identity implies that $\left(H-\lambda_{0}\right)^{-1}-\left(H_{0}-\lambda_{0}\right)^{-1} \in S_{1}$ for $\lambda_{0}<\inf \sigma(H) \leq \inf \sigma\left(H_{0}\right)$, i.e., (1.2) is true with $\mathcal{H}=H, \mathcal{H}_{0}=H_{0}$, and $\gamma=1$; consequently, the $\operatorname{SSF} \xi\left(\cdot ; H, H_{0}\right)$ exists.

Our main goal in the present paper is to study the asymptotic behavior as $b \rightarrow \infty$ of the $\operatorname{SSF} \xi\left(\cdot ; H(b), H_{0}(b)\right)$. Note that the distance between the Landau levels grows linearly with $b$. We study the SSF in the scale adapted to this rate of growth. Namely, we fix a parameter $\mathcal{E} \in \mathbb{R}$ and view $\xi\left(\mathcal{E} b+\lambda ; H(b), H_{0}(b)\right)$ as a function of $\lambda \in \mathbb{R}$. We distinguish two different types of asymptotics. The first type, which we call asymptotics far from the Landau levels, concerns the case of $\mathcal{E} \in \mathbb{R}, \mathcal{E} / 2 \notin \mathbb{Z}_{+}$. The second asymptotic regime, called asymptotics of the SSF near a Landau level, occurs when $\mathcal{E}=2 q_{0}, q_{0} \in \mathbb{Z}_{+}$. In both cases, the convergence of $\xi\left(\mathcal{E} b+\lambda ; H(b), H_{0}(b)\right)$ to its limit value is in $L_{\text {loc }}^{\infty}$ with respect to the variable $\lambda$.

The paper is organized as follows. $\S 2$ is devoted to the formulation of the main results and brief comments on them. In $\S 3$ we recall some basic properties of compact linear operators, as well as the representation formula for the SSF due to F. Gesztesy and K. Makarov [15]. Various auxiliary results utilized further in the proofs of the main ones are established in $\S 4$. The proof of Theorems 2.1 and 2.4 containing SSF asymptotics of order $\sqrt{b}$, can be found in $\S 5$. The properties of the limiting quantity that occurs in the asymptotic relations established in Theorem $[2.3$ are investigated in $\S 6$. $\S 7$ is devoted to asymptotic trace formulas for compact operators of Birman-Schwinger type. $\S 8$ contains the proof of Theorem [2.3 dealing with the case where the asymptotics of the SSF near a given Landau level is of order $b$. Finally, in $\S 9$, we reveal some spectral properties of the 
Landau Hamiltonian (see (4.1)). In particular, we prove the unitary equivalence of the Toeplitz operators corresponding to different Landau levels.

\section{§2. MAin Results}

2.1. Formulation of the main results. In this subsection we formulate our main results as Theorems 2.12.4 In our first theorem we treat the asymptotics of $\xi(\cdot ; H(b)$, $\left.H_{0}(b)\right)$ far from the Landau levels.

Theorem 2.1. Suppose (1.5) is fulfilled. Assume that $\mathcal{E} \in(0, \infty) \backslash 2 \mathbb{Z}_{+}$, and let $\Delta \subset \mathbb{R}$ be a bounded interval. Then

$$
\begin{aligned}
& \operatorname{ess} \sup _{\lambda \in \Delta}\left|\xi\left(\mathcal{E} b+\lambda ; H(b), H_{0}(b)\right)-\frac{b^{1 / 2}}{4 \pi^{2}} \sum_{q=0}^{[\mathcal{E} / 2]}(\mathcal{E}-2 q)^{-1 / 2} \int_{\mathbb{R}^{3}} V(\mathbf{x}) d \mathbf{x}\right|=O(1), \\
& b \rightarrow \infty,
\end{aligned}
$$

where $[\mathcal{E} / 2]$ denotes the integral part of the real number $\mathcal{E} / 2$.

The proof of Theorem 2.1 is contained in Subsection 5.2

The following two theorems concern the asymptotics of the SSF near a Landau level. In order to formulate our Theorem [2.3 we introduce the following family of selfadjoint operators in $L^{2}\left(\mathbb{R}, d x_{3}\right)$ :

$$
\chi_{0}:=-d^{2} / d x_{3}^{2}, \quad \chi\left(X_{\perp}\right):=\chi_{0}+V\left(X_{\perp}, \cdot\right), \quad X_{\perp} \in \mathbb{R}^{2},
$$

which are defined on the Sobolev space $\mathrm{H}^{2}(\mathbb{R})$, and depend on the parameter $X_{\perp} \in \mathbb{R}^{2}$. Then (1.5) easily implies that $\left(\chi\left(X_{\perp}\right)-\lambda_{0}\right)^{-1}-\left(\chi_{0}-\lambda_{0}\right)^{-1} \in S_{1}$ for each $X_{\perp} \in$ $\mathbb{R}^{2}$ and $\lambda_{0}<\inf \sigma\left(\chi\left(X_{\perp}\right)\right) \cup \sigma\left(\chi_{0}\right)$. Hence, the $\operatorname{SSF} \xi\left(\cdot ; \chi\left(X_{\perp}\right), \chi_{0}\right)$ is well defined as an element of $L^{1}\left(\left(\lambda_{0}, \infty\right),\left(\lambda-\lambda_{0}\right)^{-2} d \lambda\right)$. It is well known that under the assumption (1.5) $\xi\left(\cdot ; \chi\left(X_{\perp}\right), \chi_{0}\right)$ can be chosen to be continuous for $\lambda>0$. For $\lambda<0$, we choose $\xi\left(\cdot ; \chi\left(X_{\perp}\right), \chi_{0}\right)$ to be right continuous.

Proposition 2.2. For all $\lambda \neq 0$, we have $\xi\left(\lambda ; \chi(\cdot), \chi_{0}\right) \in L^{1}\left(\mathbb{R}^{2}, d X_{\perp}\right)$. Moreover, the integral $\int_{\mathbb{R}^{2}} \xi\left(\lambda ; \chi\left(X_{\perp}\right), \chi_{0}\right) d X_{\perp}$ is continuous in $\lambda>0$. This integral is continuous in $\lambda$ at $\lambda=\lambda_{0}<0$ if and only if

$$
\left|\left\{X_{\perp} \in \mathbb{R}^{2} \mid \lambda \in \sigma\left(\chi\left(X_{\perp}\right)\right)\right\}\right|=0,
$$

where $|\cdot|$ denotes the Lebesgue measure in $\mathbb{R}^{2}$.

The proof of Proposition 2.2 is given in $\S 6$.

Theorem 2.3. Assume that (1.5) is fulfilled, and let $q_{0} \in \mathbb{Z}_{+}$. Then the following statements are true.

(i) For any compact interval $\Delta_{1} \subset(0, \infty)$, we have

$$
\begin{aligned}
& \operatorname{ess}_{\sup _{\lambda \in \Delta_{1}}}\left|b^{-1} \xi\left(2 b q_{0}+\lambda ; H(b), H_{0}(b)\right)-\frac{1}{2 \pi} \int_{\mathbb{R}^{2}} \xi\left(\lambda ; \chi\left(X_{\perp}\right), \chi_{0}\right) d X_{\perp}\right| \rightarrow 0, \\
& b \rightarrow \infty \text {. }
\end{aligned}
$$


(ii) For any compact interval $\Delta_{2} \subset(-\infty, 0)$, we have

$$
\begin{aligned}
\limsup _{b \rightarrow \infty} \operatorname{ess}_{\sup _{\lambda \in \Delta_{2}}\{}\left\{b^{-1} \xi\left(2 b q_{0}+\lambda ; H(b), H_{0}(b)\right)\right. \\
\left.-\frac{1}{2 \pi} \lim _{\epsilon \rightarrow+0} \int_{\mathbb{R}^{2}} \xi\left(\lambda-\epsilon ; \chi\left(X_{\perp}\right), \chi_{0}\right) d X_{\perp}\right\} \leq 0, \\
\liminf _{b \rightarrow \infty} \operatorname{essinf}_{\lambda \in \Delta_{2}}\left\{b^{-1} \xi\left(2 b q_{0}+\lambda ; H(b), H_{0}(b)\right)\right. \\
\left.-\frac{1}{2 \pi} \lim _{\epsilon \rightarrow+0} \int_{\mathbb{R}^{2}} \xi\left(\lambda+\epsilon ; \chi\left(X_{\perp}\right), \chi_{0}\right) d X_{\perp}\right\} \geq 0 .
\end{aligned}
$$

The proof of Theorem 2.3 can be found in $\S 8$. In particular, from Theorem 2.3(ii) it follows that if the integral $\int_{\mathbb{R}^{2}} \xi\left(\lambda ; \chi\left(X_{\perp}\right), \chi_{0}\right) d X_{\perp}$ is continuous on a compact interval $\Delta_{1} \subset(-\infty, 0)$, then (2.2) is true.

Denote

$$
\Lambda:=\min _{X_{\perp} \in \mathbb{R}^{2}} \inf \sigma\left(\chi\left(X_{\perp}\right)\right) .
$$

Evidently, $\Lambda \in\left[-C_{0}, 0\right]$, and $\Lambda=0$ for nonnegative $V$. By (1.3), we have $\xi\left(\lambda ; \chi\left(X_{\perp}\right), \chi_{0}\right)$ $=0$ for all $X_{\perp}$ if $\lambda<\Lambda$. Thus, the integrals in (2.3) and (2.4) vanish if $\lambda<\Lambda$. In this case there exists a more precise version of (2.3) $-(2.4)$ contained in our last theorem.

Theorem 2.4. Let (1.5) be fulfilled. Assume that the partial derivatives of $\left\langle x_{3}\right\rangle^{m_{3}} V$ with respect to the variables $X_{\perp} \in \mathbb{R}^{2}$ exist and are uniformly bounded in $\mathbb{R}^{3}$. Let $q_{0} \in \mathbb{Z}_{+}$, and let $\Delta \subset(-\infty, \Lambda)$ be a compact interval. Then

$$
\begin{aligned}
\operatorname{ess~sup}_{\lambda \in \Delta} \mid & b^{-1 / 2} \xi\left(2 b q_{0}+\lambda ; H(b), H_{0}(b)\right) \\
& -\frac{1}{4 \pi^{2}} \sum_{q=0}^{q_{0}-1}\left(2\left(q_{0}-q\right)\right)^{-1 / 2} \int_{\mathbb{R}^{3}} V(\mathbf{x}) d \mathbf{x} \mid \rightarrow 0, \quad b \rightarrow \infty .
\end{aligned}
$$

Remark. Here and in the sequel we set the sum $\sum_{q=0}^{q_{0}-1}$ equal to zero if $q_{0}=0$.

The proof of Theorem 2.4 is contained in Subsection 5.3 .

Note that for $q_{0}=0$ the above theorem is trivial. Indeed, in this case the sum over $q$ vanishes. On the other hand, we have

$$
\Lambda=\lim _{b \rightarrow \infty} \inf \sigma(H(b)) ;
$$

this follows from [3, Theorem 5.8]. Thus, by (1.3), for all sufficiently large $b>0$ the SSF $\xi\left(2 b q_{0}+\lambda ; H(b), H_{0}(b)\right)$ in (2.5) also vanishes.

Remark. It can be shown that, for $\lambda>\Lambda$, a sufficient condition for the limit coefficient in (2.2) to be nonzero is that $V$ should be of definite sign. In this case the coefficient $\int_{\mathbb{R}^{2}} \xi\left(\lambda ; \chi\left(X_{\perp}\right), \chi_{0}\right) d X_{\perp}$ has the same sign as $V$.

2.2. Continuity of the SSF. As a by-product of our construction, we obtain a result on the continuity of the SSF. This result might be of independent interest, so we have decided to include it in the paper.

Proposition 2.5. Assume that (1.5) is true. Then the SSF $\xi\left(E ; H(b), H_{0}(b)\right)$ is bounded on every compact subset of $\mathbb{R} \backslash 2 b \mathbb{Z}_{+}$and continuous on $\mathbb{R} \backslash\left(\sigma_{p}(H(b)) \cup 2 b \mathbb{Z}_{+}\right)$, where $\sigma_{p}(H(b))$ denotes the set of eigenvalues of the operator $H(b)$.

Note that $H(b)$ may possess not only isolated negative eigenvalues but also eigenvalues embedded in the continuous spectrum $[0, \infty)$ (see [3, Theorem 5.1]). The following proposition concerns the location of the eigenvalues of $\sigma_{p}(H(b))$. 
Proposition 2.6. Assume that (1.5) is true. Then

$$
\sigma_{p}(H(b)) \subset \bigcup_{q=0}^{\infty}\left[2 b q-C\left(m_{3}\right)^{2} C_{0}^{2}, 2 b q+C\left(m_{3}\right)^{2} C_{0}^{2}\right],
$$

where $C_{0}$ is the constant occurring in (1.5) and $C\left(m_{3}\right)=(1 / 2) \int_{\mathbb{R}}\langle x\rangle^{-m_{3}} d x$.

Remark. Generically, the only possible accumulation points for the eigenvalues of $H(b)$ are the Landau levels $2 b q, q \in \mathbb{Z}_{+}$(see [3, Theorem 4.7] and [14 Theorem 3.5.3(iii)]). We shall not use this fact here.

2.3. Comments. In this subsection we comment briefly on our main results and compare them with the results existing in the literature.

- Generically, the asymptotic relations (2.1), (2.2), and (2.5) can be unified into a single formula. Indeed, it is well known (see, e.g., [1]) that

$$
\lim _{E \rightarrow \infty} E^{1 / 2} \xi\left(E ; \chi\left(X_{\perp}\right), \chi_{0}\right)=\frac{1}{2 \pi} \int_{\mathbb{R}} V\left(X_{\perp}, x_{3}\right) d x_{3}, \quad X_{\perp} \in \mathbb{R}^{2} .
$$

Thus, each of relations (2.1) with $0<\mathcal{E} / 2 \notin \mathbb{Z}_{+}$, or (2.2)-(2.5) with $2 q_{0}=\mathcal{E}, q_{0} \in \mathbb{Z}_{+}$, implies that

$$
\begin{aligned}
\xi(\mathcal{E} b+ & \left.\lambda ; H(b), H_{0}(b)\right) \\
& =\frac{b}{2 \pi} \sum_{q=0}^{[\mathcal{E} / 2]} \int_{\mathbb{R}^{2}} \xi\left(b(\mathcal{E}-2 q)+\lambda ; \chi\left(X_{\perp}\right), \chi_{0}\right) d X_{\perp}(1+o(1)), \quad b \rightarrow \infty,
\end{aligned}
$$

in $L_{\mathrm{loc}}^{\infty}(\mathbb{R} \backslash\{0\})$. In its turn, formula (2.7) can be rewritten as

$$
\begin{aligned}
\xi(\mathcal{E} b & \left.+\lambda ; H(b), H_{0}(b)\right) \\
& =\int_{\mathbb{R}} \int_{\mathbb{R}^{2}} \xi\left(\mathcal{E} b+\lambda-s ; \chi\left(X_{\perp}\right), \chi_{0}\right) d X_{\perp} d \nu_{b}(s)(1+o(1)), \quad b \rightarrow \infty,
\end{aligned}
$$

where

$$
\nu_{b}(s):=\frac{b}{2 \pi} \sum_{q=0}^{\infty} \Theta(s-2 b q), \quad s \in \mathbb{R}
$$

and

$$
\Theta(s):= \begin{cases}0 & \text { if } s \leq 0 \\ 1 & \text { if } s>0\end{cases}
$$

is the Heaviside function. It is well known that $\nu$ is the integrated density of states for the two-dimensional Landau Hamiltonian (see (4.1)).

- By (1.3), for almost every $\lambda<0$ we have $\xi\left(\lambda ; H(b), H_{0}\right)=-N(\lambda ; H(b))$. In the case where $\lambda<0$ is fixed, the asymptotics as $b \rightarrow \infty$ of the counting function $N(\lambda ; H(b))$ was investigated in 25 under far less restrictive assumptions on $V$ than in Theorems 2.12.4. The asymptotic properties as $\lambda \uparrow 0$ and as $\lambda \downarrow \Lambda$ of the asymptotic coefficient $-\frac{1}{2 \pi} \int_{\mathbb{R}^{2}} N\left(\lambda ; \chi\left(X_{\perp}\right)\right) d X_{\perp}$ (which appears in (2.3) and (2.4) in the case of a negative perturbation) were studied in [26].

- As mentioned in the Introduction, on the absolutely continuous spectrum of $H_{0}(b)$ the SSF is related to the scattering determinant by the Birman-Krein formula (1.4). The existence and completeness of the wave operators for the pair $\left((i \nabla+\mathbf{A})^{2}+U,(i \nabla+\mathbf{A})^{2}\right)$ was established in [3] for quite general electromagnetic potentials $(\mathbf{A}, U)$ including the case of constant magnetic fields. More recent results on the scattering theory for the pair $\left(H_{0}(b)+V, H_{0}(b)\right)$ can be found in the monograph [14]. As far as the authors are informed, the asymptotics as $b \rightarrow \infty$ of the scattering phase $\arg \operatorname{det} S\left(\lambda ; H(b), H_{0}(b)\right)$ has not been presented in the literature before. 
- Finally, we would like to mention several possible extensions of our results.

We believe that the assumptions about the continuity and boundedness of $V$ and its derivatives can be lifted by using an appropriate approximation procedure. In particular, we expect that some local integrable singularities can be allowed. We do not include this approximation procedure in the present paper, in order to avoid an unreasonable growth of its size.

Furthermore, it is natural to try to obtain an optimal remainder estimate in (2.2) and (2.5), in the cases where such an estimate exists. For this, hopefully, the theory of the pseudodifferential operators with operator-valued symbols developed in [5], [18], and [12] could be useful.

\section{§3. Representation of the SSF AS AN AVERAGED INDEX OF PROJECTiONS OF COMPACT OPERATORS}

In this section we recall the representation formula for the SSF (see formula (3.15) below) in terms of an integral of an index for a Fredholm pair of selfadjoint spectral projections. This formula was obtained in [15] for the case of trace class perturbation and was generalized in [23] to the case of relatively trace class perturbations. The formula generalizes the earlier results of [21], 30] and can be regarded as a far-reaching extension of the Birman-Schwinger principle (see [7]).

In order to write down this formula, we need to recall some basic properties of compact operators and the notion of the index of a pair of projections.

3.1. Basic properties of compact linear operators. We denote by $S_{\infty}$ the class of compact linear operators acting in a fixed Hilbert space. Let $T=T^{*} \in S_{\infty}$, and let $E_{T}(I)$ denote the spectral projection of $T$ associated with the interval $I \subset \mathbb{R}$. For $s>0$, we set

$$
n_{ \pm}(s ; T):=\operatorname{rank} E_{ \pm T}((s, \infty)) .
$$

For an arbitrary (not necessarily selfadjoint) operator $T \in S_{\infty}$, put

$$
n_{*}(s ; T):=n_{+}\left(s^{2} ; T^{*} T\right), \quad s>0 .
$$

If $T=T^{*}$, then, obviously,

$$
n_{*}(s ; T)=n_{+}(s, T)+n_{-}(s ; T), \quad s>0 .
$$

Moreover, if $T_{j}=T_{j}^{*} \in S_{\infty}, j=1,2$, then the Weyl inequalities

$$
n_{ \pm}\left(s_{1}+s_{2}, T_{1}+T_{2}\right) \leq n_{ \pm}\left(s_{1}, T_{1}\right)+n_{ \pm}\left(s_{2}, T_{2}\right)
$$

are fulfilled for all $s_{1}, s_{2}>0$.

Next, we denote by $S_{p}, p \in(0, \infty)$, the Schatten-von Neumann class of compact operators for which the functional $\|T\|_{S_{p}}:=\left(p \int_{0}^{\infty} s^{p-1} n_{*}(s ; T) d s\right)^{\frac{1}{p}}$ is finite. If $T \in S_{p}$, $p \in(0, \infty)$, then the elementary inequality

$$
n_{*}(s ; T) \leq s^{-p}\|T\|_{S_{p}}^{p}
$$

is true for every $s>0$. If $T=T^{*} \in S_{p}, p \in(0, \infty)$, then (3.1) and (3.2) imply

$$
n_{ \pm}(s ; T) \leq s^{-p}\|T\|_{S_{p}}^{p}, \quad s>0 .
$$

Finally, we define the selfadjoint operators $\operatorname{Re} T:=\frac{1}{2}\left(T+T^{*}\right)$ and $\operatorname{Im} T:=\frac{1}{2 i}\left(T-T^{*}\right)$. Evidently,

$$
n_{ \pm}(s ; \operatorname{Re} T) \leq 2 n_{*}(s ; T), \quad n_{ \pm}(s ; \operatorname{Im} T) \leq 2 n_{*}(s ; T) .
$$


3.2. Index of a pair of projections. A pair of orthogonal projections $P, Q$ in a Hilbert space is said to be Fredholm if

$$
\{1,-1\} \cap \sigma_{\text {ess }}(P-Q)=\varnothing .
$$

In particular, if $P-Q$ is compact, then the pair $P, Q$ is Fredholm. The index of a Fredholm pair is given by the formula

$$
\text { index }(P, Q)=\operatorname{dim} \operatorname{Ker}(P-Q-I)-\operatorname{dim} \operatorname{Ker}(P-Q+I) .
$$

In particular, if $P-Q \in S_{1}$, then $\operatorname{index}(P, Q)=\operatorname{Tr}(P-Q)$. Clearly,

$$
\operatorname{index}(P, Q)=-\operatorname{index}(Q, P)=-\operatorname{index}(I-P, I-Q) \text {. }
$$

If both $P, Q$ and $Q, R$ are Fredholm pairs and either $P-Q$ or $Q-R$ is compact, then the pair $P, R$ is also Fredholm, and the following "chain rule" holds true:

$$
\text { index }(P, R)=\operatorname{index}(P, Q)+\operatorname{index}(Q, R) .
$$

See, e.g., [4 for the proof of the last statement and the details.

Suppose $M$ and $\widetilde{M}$ are bounded selfadjoint operators. If the spectral projections $E_{M}((-\infty, 0)), E_{\widetilde{M}}((-\infty, 0))$ form a Fredholm pair, we shall use the shorthand notation

$$
\operatorname{ind}(\widetilde{M}, M):=\operatorname{index}\left(E_{\widetilde{M}}((-\infty, 0)), E_{M}((-\infty, 0))\right) .
$$

Mostly, we shall use this notation if $\widetilde{M}=M+A$, where $A$ is a compact selfadjoint operator and $M$ is a bounded selfadjoint operator such that 0 is not in the essential spectrum of $M$. In this case, representing the spectral projections by Riesz integrals and using the resolvent identity, we easily see that the difference $E_{\widetilde{M}}((-\infty, 0))-E_{M}((-\infty, 0))$ is compact, and therefore the above pair of spectral projections is Fredholm.

Below we list the properties of ind that we shall need in the paper.

(a) If $A \in S_{1}$ and 0 is not in the essential spectrum of $M$ (and hence of $M+A$ ), then

$$
\operatorname{ind}(M+A, M)=\lim _{\epsilon \rightarrow+0} \xi(-\epsilon ; M, M+A) .
$$

This follows from the trace formula (1.1) after approximation of the characteristic function of the interval $(-\infty, 0)$ by smooth functions.

(b) If $0 \leq A \in S_{\infty}$, and 0 is not in the spectrum of $M$, then there exists a simple Birman-Schwinger type formula for computing ind $(M+A, M)$ :

$$
\operatorname{ind}(M+A, M)=-\sum_{s \in[0,1]} \operatorname{dim} \operatorname{Ker}(M+s A)=-\operatorname{rank} E_{A^{1 / 2} M^{-1} A^{1 / 2}}((-\infty,-1])
$$

see [15. Corollary 4.8] and 23] Lemma 5.2] for the proof.

(c) There is a stability result for ind (see, e.g., [15, Theorem 3.12] for the proof):

(d) If $A$ is a finite rank operator, then

$$
-\operatorname{rank} A_{+} \leq \operatorname{ind}(M+A, M) \leq \operatorname{rank} A_{-},
$$

where $A_{ \pm}=\frac{1}{2}(|A| \pm A)$. This follows from property (a) and the similar bound for the SSF (see, e.g., 31]).

(e) If $\widetilde{M} \geq M$, then $\operatorname{ind}(\widetilde{M}, M) \leq 0$.

Proof. It suffices to prove that

$$
\operatorname{Ker}\left(E_{\widetilde{M}}((-\infty, 0))-E_{M}((-\infty, 0))-I\right)=\{0\} .
$$


Suppose to the contrary that $\left(E_{\widetilde{M}}((-\infty, 0))-E_{M}((-\infty, 0))-I\right) \psi=0$ for a vector $\psi \neq 0$; then $E_{\widetilde{M}}((-\infty, 0)) \psi=\psi$ and $E_{M}((-\infty, 0)) \psi=0$. It follows that $(\widetilde{M} \psi, \psi)<0$ and $(M \psi, \psi) \geq 0$, a contradiction.

(f) Let $M$ be a bounded selfadjoint operator such that $[-a, a] \subset \rho(M)$ for some $a>0$. Let $A_{0}$ and $A$ be compact selfadjoint operators and suppose that $\|A\| \leq a$. Then

$$
\operatorname{ind}\left(M+A_{0}+a, M+a\right) \leq \operatorname{ind}\left(M+A_{0}+A, M\right) \leq \operatorname{ind}\left(M+A_{0}-a, M-a\right) .
$$

Proof. By the chain rule,

$$
\operatorname{ind}\left(M+A_{0}+A, M\right)=\operatorname{ind}\left(M+A_{0}+A, M+A_{0}-a\right)+\operatorname{ind}\left(M+A_{0}-a, M\right) .
$$

By property (e), ind $\left(M+A_{0}+A, M+A_{0}-a\right) \leq 0$. This gives the second of the two required inequalities. The first can be obtained in a similar way.

(g) Combining properties (d) and (f), we obtain the following estimate. Let $M$ be a bounded selfadjoint operator such that $[-a, a] \subset \rho(M)$ for some $a>0$. Let $A_{0}$ and $A$ be compact selfadjoint operators. Then

$$
\begin{aligned}
& \operatorname{ind}\left(M+A_{0}+a, M+a\right)-n_{+}(a, A) \\
& \quad \leq \operatorname{ind}\left(M+A_{0}+A, M\right) \leq \operatorname{ind}\left(M+A_{0}-a, M-a\right)+n_{-}(a, A) .
\end{aligned}
$$

3.3. Representation of the SSF. Now we are ready to describe the required representation for the SSF.

Let $\mathcal{H}$ and $\mathcal{H}_{0}$ be two lower bounded selfadjoint operators acting in the same Hilbert space. Let $\lambda_{0}<\inf \sigma(\mathcal{H}) \cup \sigma\left(\mathcal{H}_{0}\right)$. First, assume that (1.2) is true for some $\gamma>0$. Next, let

$$
\mathcal{V}:=\left(\mathcal{H}-\mathcal{H}_{0}\right)=\mathcal{K}^{*} \mathcal{J} \mathcal{K},
$$

where $\mathcal{J}$ is a bounded selfadjoint operator with a bounded inverse, and

$$
\mathcal{K}\left(\mathcal{H}_{0}-\lambda_{0}\right)^{-1 / 2} \in S_{\infty}
$$

Finally, suppose that

$$
\mathcal{K}\left(\mathcal{H}_{0}-\lambda_{0}\right)^{-\gamma^{\prime}} \in S_{2}
$$

for some $\gamma^{\prime}>0$. For $z \in \mathbb{C}$ with $\operatorname{Im} z>0$, we set $\mathcal{T}(z):=\mathcal{K}\left(\mathcal{H}_{0}-z\right)^{-1} \mathcal{K}^{*}$.

Lemma 3.1 (see 8]). Let (3.12)-3.14) be fulfilled. Then for almost every $E \in \mathbb{R}$ the operator norm limit $\mathcal{T}(E+i 0):=\mathrm{n}-\lim _{\delta \downarrow 0} \mathcal{T}(E+i \delta)$ exists, and by (3.13) we have $\mathcal{T}(E+i 0) \in S_{\infty}$. Moreover, $\operatorname{Im} \mathcal{T}(E+i 0) \in S_{1}$.

Theorem 3.2 (see [15, 23]). Let (1.2) and (3.12)-(3.14) be fulfilled. Then for almost every $E \in \mathbb{R}$ we have

$$
\xi\left(E ; \mathcal{H}, \mathcal{H}_{0}\right)=\int_{-\infty}^{\infty} \operatorname{ind}\left(\mathcal{J}^{-1}+\operatorname{Re} \mathcal{T}(E+i 0)+t \operatorname{Im} \mathcal{T}(E+i 0), \mathcal{J}^{-1}\right) d \mu(t),
$$

where $d \mu(t)=\pi^{-1}\left(1+t^{2}\right)^{-1} d t$.

The representation (3.15) was found in 15 for the case of the trace class $\mathcal{V}$. A generalization for relatively trace class perturbations was proved in 23 .

Let us comment on the convergence of the integral in (3.15). Below we mimic the proof of [21, Lemma 2.1]. Choose $s>0$ sufficiently small, so that $[-s, s]$ does not contain the spectrum of $\mathcal{J}^{-1}$. Using property (g) of ind, we see that

$$
\begin{gathered}
\left|\operatorname{ind}\left(\mathcal{J}^{-1}+\operatorname{Re} \mathcal{T}(E+i 0)+t \operatorname{Im} \mathcal{T}(E+i 0), \mathcal{J}^{-1}\right)\right| \\
\leq n_{*}(s, \operatorname{Re} \mathcal{T}(E+i 0)+t \operatorname{Im} \mathcal{T}(E+i 0)) .
\end{gathered}
$$


Applying Weyl's inequality (3.3), for any $s_{1}, s_{2} \in(0,1)$ with $s_{1}+s_{2}=s$ we obtain

$$
\begin{aligned}
\int_{-\infty}^{\infty} & n_{*}(s, \operatorname{Re} \mathcal{T}(E+i 0)+t \operatorname{Im} \mathcal{T}(E+i 0)) d \mu(t) \\
\quad \leq & n_{*}\left(s_{1}, \operatorname{Re} \mathcal{T}(E+i 0)\right)+\int_{0}^{\infty} n_{*}\left(s_{2}, t \operatorname{Im} \mathcal{T}(E+i 0)\right) d \mu(t) \\
\quad \leq & n_{*}\left(s_{1}, \operatorname{Re} \mathcal{T}(E+i 0)\right)+\frac{1}{\pi s_{2}}\|\operatorname{Im} \mathcal{T}(E+i 0)\|_{S_{1}} .
\end{aligned}
$$

This proves the absolute convergence of the integral in (3.15) and provides a bound, which will be used in the sequel.

Now, suppose that $V$ satisfies (1.5). Then relations (3.12)-(3.14) are true with $\mathcal{V}=V$, $\mathcal{H}_{0}=H_{0}, \mathcal{K}=|V|^{1 / 2}, \mathcal{J}=\operatorname{sgn} V$, and $\gamma=\gamma^{\prime}=1$. For $z \in \mathbb{C}, \operatorname{Im} z>0$, we set $T(z):=|V|^{1 / 2}\left(H_{0}-z\right)^{-1}|V|^{1 / 2}$. By Lemma 3.1 for almost every $E \in \mathbb{R}$ the operator norm limit

$$
T(E+i 0):=\mathrm{n}-\lim _{\delta \downarrow} T(E+i \delta)
$$

exists, and

$$
0 \leq \operatorname{Im} T(E+i 0) \in S_{1} .
$$

From Lemma 4.2 below it follows that the limit in (3.18) exists and relation (3.19) is valid for every $E \notin 2 b \mathbb{Z}_{+}$. Denote

$$
A(E)=\operatorname{Re} T(E+i 0), \quad B(E)=\operatorname{Im} T(E+i 0), \quad J=\operatorname{sgn} V .
$$

Then (3.15) becomes

$$
\xi\left(E ; H(b), H_{0}(b)\right)=\int_{-\infty}^{\infty} \operatorname{ind}(J+A(E)+t B(E), J) d \mu(t) \quad \text { for a.e. } E \in \mathbb{R},
$$

the right-hand side being well defined for every $E \in \mathbb{R} \backslash 2 b \mathbb{Z}_{+}$.

\section{§4. Preliminary uniform estimates}

In Subsection 4.1, we obtain estimates of the spectrum of the sandwiched resolvent $T(E+i 0)$, which will be essential for the proofs of Theorems 2.1 2.3. and 2.4 in $\S \S 5$ and 8. Moreover, these estimates allow us to prove Propositions 2.5] and 2.6. which is done in Subsection 4.2.

4.1. Estimates for the sandwiched resolvent. We recall (see, e.g., 3]) the formula for the resolvent of $H_{0}(b)$ :

$$
\left(H_{0}(b)-z\right)^{-1}=\sum_{q=0}^{\infty} p_{q} \otimes r(z-2 b q), \quad \operatorname{Im} z>0 .
$$

Here $p_{q}$ is the orthogonal projection onto the eigenspace corresponding to the Landau level $2 b q$ of the two-dimensional magnetic Hamiltonian

$$
h(b):=\left(i \frac{\partial}{\partial x_{1}}-\frac{b x_{2}}{2}\right)^{2}+\left(i \frac{\partial}{\partial x_{2}}+\frac{b x_{1}}{2}\right)^{2}-b,
$$

and $r(z)$ is the resolvent of $-d^{2} / d x_{3}^{2}$ in $L^{2}\left(\mathbb{R}, d x_{3}\right)$. By the assumption (1.5) on $V$, we can write $|V(\mathbf{x})|=M(\mathbf{x})\left\langle X_{\perp}\right\rangle^{-m_{\perp}}\left\langle x_{3}\right\rangle^{-m_{3}}$, where $0 \leq M \in L^{\infty}\left(\mathbb{R}^{3}\right)$. 
For $z \in \mathbb{C}_{+}:=\{z \in \mathbb{C} \mid \operatorname{Im} z>0\}$, consider the sandwiched resolvent

$$
\begin{aligned}
T(z) & =\sum_{q=0}^{\infty} T_{q}(z), \\
T_{q}(z) & =M^{1 / 2}\left(\left(\left\langle X_{\perp}\right\rangle^{-m_{\perp} / 2} p_{q}\left\langle X_{\perp}\right\rangle^{-m_{\perp} / 2}\right) \otimes t(z-2 b q)\right) M^{1 / 2}, \\
t(z) & =\left\langle x_{3}\right\rangle^{-m_{3} / 2} r(z)\left\langle x_{3}\right\rangle^{-m_{3} / 2} .
\end{aligned}
$$

Bounds on $t(z)$. Recall that $m_{3}>1$. Under this assumption, it is well known (and can be seen immediately from the explicit form of the integral kernel of $r(z)$ ) that for $z \in \mathbb{C}_{+}$ the operator $t(z)$ belongs to the Hilbert-Schmidt class $S_{2}$, is continuous in $z \in \overline{\mathbb{C}_{+}} \backslash\{0\}$ in the Hilbert-Schmidt norm, and obeys the bound

$$
\|t(E+i 0)\|_{S_{2}} \leq \frac{C\left(m_{3}\right)}{\sqrt{|E|}}, \quad E \in \mathbb{R} \backslash\{0\},
$$

where $C\left(m_{3}\right)=(1 / 2) \int_{\mathbb{R}}\langle x\rangle^{-m_{3}} d x$. It is less evident that similar properties hold true in the trace class $S_{1}$. This can be seen as follows. By the spectral theorem, we can write

$$
t(z)=\int_{0}^{\infty} F(\nu)^{*} F(\nu) \frac{d \nu}{\nu-z}, \quad z \in \mathbb{C}_{+},
$$

where $F(\nu): L^{2}(\mathbb{R}) \rightarrow \mathbb{C}^{2}$ is given by

$$
\begin{aligned}
F(\nu): u(x) \mapsto & \left(\frac{1}{2 \sqrt{\pi}} \nu^{-1 / 4} \int_{-\infty}^{\infty} e^{+i \sqrt{\nu} x}\langle x\rangle^{-m_{3} / 2} u(x) d x,\right. \\
& \left.\frac{1}{2 \sqrt{\pi}} \nu^{-1 / 4} \int_{-\infty}^{\infty} e^{-i \sqrt{\nu} x}\langle x\rangle^{-m_{3} / 2} u(x) d x\right) .
\end{aligned}
$$

It is easily seen that $F(\nu)$ belongs to the Hilbert-Schmidt class $S_{2}$, and is Hölder continuous in $\nu$ in the Hilbert-Schmidt norm. It follows that, for any $z \in \mathbb{C}_{+}$, the operator $t(z)$ belongs to the trace class, is continuous in $z \in \overline{\mathbb{C}_{+}} \backslash\{0\}$ in the trace norm, and satisfies the inequality

$$
\|t(E+i 0)\|_{S_{1}} \leq \frac{C}{\sqrt{|E|}}\left(1+E_{+}^{1 / 4}\right), \quad E \in \mathbb{R} \backslash\{0\} .
$$

The right-hand side in (4.4) can be replaced by $\frac{C}{\sqrt{|E|}}\left(1+E_{+}^{\delta}\right)$ for any $\delta>0$, but for our purposes it suffices to take $\delta=1 / 4$. Finally, a direct inspection of the integral kernel shows that $\operatorname{Im} t(E+i 0)$ has rank two for all $E \neq 0$, and we have the estimate

$$
\|\operatorname{Im} t(E+i 0)\|_{S_{p}}^{p} \leq C|E|^{-p / 2}
$$

for any $0<p \leq 1$.

A bound on $\left\langle X_{\perp}\right\rangle^{-m_{\perp} / 2} p_{q}\left\langle X_{\perp}\right\rangle^{-m_{\perp} / 2}$. We have an explicit formula for the integral kernel of $p_{q}$ :

$$
K_{q, b}\left(\mathbf{x}, \mathbf{x}^{\prime}\right)=\frac{b}{2 \pi} L_{q}^{(0)}\left(\frac{b\left|\mathbf{x}-\mathbf{x}^{\prime}\right|^{2}}{2}\right) \exp \left(-\frac{b}{4}\left(\left|\mathbf{x}-\mathbf{x}^{\prime}\right|^{2}+2 i\left(x_{1} x_{2}^{\prime}-x_{1}^{\prime} x_{2}\right)\right)\right)
$$

(see, e.g., [20] or [27. Subsection 2.3.2]), where the Laguerre polynomials $L_{q}^{(0)}$ are defined as in (9.7) below. Note that $K_{q, b}(\mathbf{x}, \mathbf{x})=\frac{b}{2 \pi}$ for each $q \in \mathbb{Z}_{+}$and $\mathbf{x} \in \mathbb{R}^{2}$. Using this 
fact, we immediately obtain

$$
\begin{aligned}
& \left\|\left\langle X_{\perp}\right\rangle^{-m_{3}} p_{q}\right\|_{S_{2}}^{2}=\left\|\left\langle X_{\perp}\right\rangle^{-m_{\perp} / 2} p_{q}\left\langle X_{\perp}\right\rangle^{-m_{\perp} / 2}\right\|_{S_{1}} \\
& \quad=\operatorname{Tr}\left(\left\langle X_{\perp}\right\rangle^{-m_{\perp} / 2} p_{q}\left\langle X_{\perp}\right\rangle^{-m_{\perp} / 2}\right)=\frac{b}{2 \pi} \int_{\mathbb{R}^{2}}\left\langle X_{\perp}\right\rangle^{-m_{\perp}} d X_{\perp} \\
& \quad=C b .
\end{aligned}
$$

Later on, we shall prove a stronger bound (see Lemma 8.1).

Bounds on $T_{q}(z)$. Putting together the above bounds in (4.2), we obtain the following statement.

Lemma 4.1. For any $z \in \mathbb{C}_{+}$, the operator $T_{q}(z)$ belongs to the trace class $S_{1}$ and depends continuously on $z \in \overline{\mathbb{C}_{+}} \backslash\{2 b q\}$ in the trace class norm. Moreover,

$$
\begin{array}{cc}
\left\|T_{q}(E+i 0)\right\| \leq \frac{C_{1}}{|E-2 b q|^{1 / 2}}, & E \in \mathbb{R} \backslash\{2 b q\}, \\
\left\|T_{q}(E+i 0)\right\|_{S_{1}} \leq \frac{C_{2} b}{|E-2 b q|^{1 / 2}}\left(1+|E-2 b q|^{1 / 4}\right), & E \in \mathbb{R} \backslash\{2 b q\} .
\end{array}
$$

In the sequel, for $E \in \mathbb{R} \backslash\{2 b q\}$ and $\lambda \in \mathbb{R} \backslash\{0\}$, we denote

$$
\begin{aligned}
T_{q}(E) & =M^{1 / 2}\left(\left(\left\langle X_{\perp}\right\rangle^{-m_{\perp} / 2} p_{q}\left\langle X_{\perp}\right\rangle^{-m_{\perp} / 2}\right) \otimes t(E-2 b q)\right) M^{1 / 2}, \\
t(\lambda) & =\left\langle x_{3}\right\rangle^{-m_{3} / 2} r(\lambda+i 0)\left\langle x_{3}\right\rangle^{-m_{3} / 2} .
\end{aligned}
$$

Finally, we get a bound for $T(z)$.

Lemma 4.2. For any $z \in \mathbb{C}_{+}$, the operator $T(z)$ is compact, depends continuously on $z \in \overline{\mathbb{C}_{+}} \backslash 2 b \mathbb{Z}_{+}$in the operator norm, and obeys the estimate

$$
\|T(E+i 0)\| \leq \frac{C_{0} C\left(m_{3}\right)}{\operatorname{dist}\left(E, 2 b \mathbb{Z}_{+}\right)^{1 / 2}}, \quad E \in \mathbb{R} \backslash 2 b \mathbb{Z}_{+} .
$$

Moreover, for $E \in \mathbb{R} \backslash 2 b \mathbb{Z}_{+}$the operator $B(E)=T(E+i 0)$ is nonnegative, and it vanishes if $E<0$. Next, $B(E)$ belongs to the trace class $S_{1}$ and depends continuously on $E \in \mathbb{R} \backslash 2 b \mathbb{Z}_{+}$in the trace class norm. Finally, for $2 b\left(q_{0}-1\right)<E<2 b q_{0}, q_{0} \in \mathbb{Z}_{+}$, $q_{0} \geq 1$, and $r=0,1$, we have

$$
\operatorname{Tr}\left(J^{r} B(E)\right)=\frac{b}{4 \pi} \sum_{q=0}^{q_{0}-1}(E-2 b q)^{-1 / 2} \int_{\mathbb{R}^{3}}(\operatorname{sgn} V(\mathbf{x}))^{r}|V(\mathbf{x})| d \mathbf{x} .
$$

Proof. Compactness follows from the diamagnetic inequality; in fact, we have $T(z) \in S_{2}$, but we do not need this here. Next, fix $q_{0} \in \mathbb{Z}_{+}$. For $E<2 b q_{0}, E \in \mathbb{R} \backslash 2 b \mathbb{Z}_{+}$, continuity in the operator norm can be seen by representing

$$
T(E+i 0)=\sum_{q=0}^{q_{0}-1} T_{q}(E)+T_{q_{0}}^{+}(E) .
$$

Here each $T_{q}(E)$ is continuous by Lemma 4.1 and $T_{q_{0}}^{+}(E)$ is even analytic in $E \in \mathbb{C} \backslash$ $\left[2 b q_{0}, \infty\right)$ by the spectral theorem. Using (4.3), we obtain

$$
\begin{aligned}
\|T(E+i 0)\| & \leq C_{0}\left\|\sum_{q=0}^{\infty} p_{q} \otimes t(E-2 b q) \leq C_{0} \sup _{q \in \mathbb{Z}_{+}}\right\| t(E-2 b q) \\
& \leq \frac{C_{0} C\left(m_{3}\right)}{\operatorname{dist}\left(E, 2 b \mathbb{Z}_{+}\right)^{1 / 2}} .
\end{aligned}
$$


Taking the imaginary parts in (4.13) gives

$$
B(E)=\sum_{q=0}^{q_{0}-1} B_{q}(E), \quad E<2 b q_{0}, \quad E \in \mathbb{R} \backslash 2 b \mathbb{Z}_{+},
$$

where $B_{q}(E)=\operatorname{Im} T_{q}(E)$ is trace norm continuous by Lemma4.1. Note that the relations $B(E) \geq 0$ for $E \in \mathbb{R} \backslash 2 b \mathbb{Z}_{+}$, and $B(E)=0$ for $E<0$, follow easily from the definition of the operator $T(z)$ (see (4.2)).

Finally, using (4.10), (4.6) and the explicit formula for the integral kernel of $\operatorname{Im} t(\lambda)$,

$$
\left\langle x_{3}\right\rangle^{-m_{3} / 2} \frac{\cos \left(\sqrt{\lambda}\left(x_{3}-x_{3}^{\prime}\right)\right)}{2 \sqrt{\lambda}}\left\langle x_{3}^{\prime}\right\rangle^{-m_{3} / 2}, \quad x_{3}, x_{3}^{\prime} \in \mathbb{R},
$$

we obtain 4.12).

\subsection{Proof of Propositions 2.5 and 2.6.}

4.2.1. Proof of Proposition [2.5. We identify the SSF with the right-hand side of the representation (3.21) and prove its continuity on the set $\mathbb{R} \backslash\left(\sigma_{p}(H(b)) \cup 2 b \mathbb{Z}_{+}\right)$. By the stability result of [15. Theorem 3.12], the right-hand side of (3.21) is continuous in $E$ at a point $E=E_{0}$ if the following conditions are satisfied:

$$
\begin{gathered}
\lim _{E \rightarrow E_{0}}\left\|A(E)-A\left(E_{0}\right)\right\|=0, \quad \lim _{E \rightarrow E_{0}}\left\|B(E)-B\left(E_{0}\right)\right\|_{S_{1}}=0 . \\
0 \in \rho\left(J+A\left(E_{0}\right)+\tau B\left(E_{0}\right)\right) \quad \text { for some } \tau \in \mathbb{R} .
\end{gathered}
$$

The limit relations (4.14) are met by Lemma 4.2 By the analytic Fredholm alternative, 4.15) will follow from $0 \in \rho\left(J+A\left(E_{0}\right)+i B\left(E_{0}\right)\right)$ or, equivalently, from $-1 \in$ $\rho\left(J T\left(E_{0}+i 0\right)\right)$. Mimicking Agmon's "bootstrap argument" [2], we see that

$$
-1 \in \sigma\left(J T\left(E_{0}+i 0\right)\right) \Longleftrightarrow E_{0} \in \sigma_{p}(H(b))
$$

for $E_{0} \in \mathbb{R} \backslash 2 b \mathbb{Z}_{+}$. Thus, condition (4.15) holds true for all $E_{0} \in \mathbb{R} \backslash\left(\sigma_{p}(H(b)) \cup 2 b \mathbb{Z}_{+}\right)$.

Now, we prove that the right-hand side of (3.21) is bounded on any closed interval that contains no Landau levels. By (3.17), we have

$$
\left|\int_{-\infty}^{\infty} \operatorname{ind}(J+A(E)+t B(E), J) d \mu(t)\right| \leq n(1 / 3, A(E))+\frac{3}{\pi}\|B(E)\|_{S_{1}} .
$$

By Lemma 4.2 the right-hand side is bounded on any closed interval in $\mathbb{R} \backslash 2 b \mathbb{Z}_{+}$.

4.2.2. Proof of Proposition 2.6. By (4.16), the problem reduces to estimating the norm of $T(E+i 0)$. Estimate (4.11) shows that for $\operatorname{dist}\left(E, 2 b \mathbb{Z}_{+}\right)>C_{0}^{2} C\left(m_{3}\right)^{2}$ we have $\|T(E+i 0)\|<1$, whence $E \notin \sigma_{p}(H(b))$.

\section{§5. SSF ASYMPTOTICS OF THE ORDER OF $\sqrt{b}$}

In this section the asymptotic behavior of the $\operatorname{SSF} \xi\left(E ; H, H_{0}\right)$ as $b \rightarrow+\infty$ is studied in two situations. First, to prove Theorem [2.1, we consider energies far from the Landau levels, that is, $E=\mathcal{E} b+\lambda$ with $\mathcal{E} \in \mathbb{R} \backslash 2 \mathbb{Z}_{+}$. Then, to prove Theorem 2.4 we consider energies under Landau levels, that is, $E=2 q_{0} b+\lambda$ with $\lambda<\Lambda(\Lambda$ : $=$ $\left.\min _{X_{\perp} \in \mathbb{R}^{2}} \inf \sigma\left(\chi\left(X_{\perp}\right)\right)\right)$. 
5.1. An abstract lemma. First, we prove a simple lemma of abstract nature. This lemma might be of independent interest. Note that formulas similar to (5.1) can be found in [15].

Lemma 5.1. Let $A=A^{*}$ be a compact operator, let $0 \leq B \in S_{1}$, and let $J=J^{*}=J^{-1}$. Suppose that $0 \in \rho(J+A)$. Then

$$
\begin{aligned}
& \int_{-\infty}^{\infty} \operatorname{ind}(J+A+t B, J) d \mu(t) \\
& \quad=\operatorname{ind}(J+A, J)+\pi^{-1} \operatorname{Tr} \arctan \left(B^{1 / 2}(J+A)^{-1} B^{1 / 2}\right)
\end{aligned}
$$

and

$$
\begin{aligned}
& \left|\pi^{-1} \operatorname{Tr} \arctan \left(B^{1 / 2}(J+A)^{-1} B^{1 / 2}\right)-\pi^{-1} \operatorname{Tr}(J B)\right| \\
& \quad \leq \frac{1}{3}\left\|(J+A)^{-1}\right\|^{3} \operatorname{Tr} B^{3}+\left|\operatorname{Tr}\left(B J A(J+A)^{-1}\right)\right| .
\end{aligned}
$$

Proof. Using the "chain rule" (3.7) and integrating, we get

$$
\int_{-\infty}^{\infty} \operatorname{ind}(J+A+t B, J) d \mu(t)=\operatorname{ind}(J+A, J)+\int_{-\infty}^{\infty} \operatorname{ind}(J+A+t B, J+A) d \mu(t) .
$$

We split the integral on the right into the sum of integrals over $(-\infty, 0)$ and $(0, \infty)$. Using (3.9), we obtain

$$
\begin{aligned}
\int_{-\infty}^{\infty} & \operatorname{ind}(J+A+t B, J+A) d \mu(t) \\
= & \int_{0}^{\infty} \operatorname{ind}(J+A+t B, J+A) d \mu(t)+\int_{0}^{\infty} \operatorname{ind}(J+A-t B, J+A) d \mu(t) \\
= & -\int_{0}^{\infty} n_{-}\left(1, t B^{1 / 2}(J+A)^{-1} B^{1 / 2}\right) d \mu(t) \\
& +\int_{0}^{\infty} n_{+}\left(1, t B^{1 / 2}(J+A)^{-1} B^{1 / 2}\right) d \mu(t) \\
= & \pi^{-1} \operatorname{Tr} \arctan \left(B^{1 / 2}(J+A)^{-1} B^{1 / 2}\right) .
\end{aligned}
$$

This proves formula (5.1).

Now, we prove (5.2). Denoting $M=B^{1 / 2}(J+A)^{-1} B^{1 / 2}$, we get

$\operatorname{Tr} \arctan M-\operatorname{Tr} M$

$$
=\int_{0}^{\infty} \frac{n_{+}(t, M)}{1+t^{2}} d t-\int_{0}^{\infty} \frac{n_{-}(t, M)}{1+t^{2}} d t-\int_{0}^{\infty} n_{+}(t, M) d t+\int_{0}^{\infty} n_{-}(t, M) d t,
$$

whence

$$
\begin{aligned}
& |\operatorname{Tr} \arctan M-\operatorname{Tr} M| \leq \int_{0}^{\infty} \frac{t^{2} n_{*}(t, M)}{1+t^{2}} d t \leq \int_{0}^{\infty} t^{2} n_{*}(t, M) d t=\frac{1}{3}\|M\|_{S_{3}}^{3} \\
& \quad \leq \frac{1}{3}\left(\left\|B^{1 / 2}\right\|_{S_{6}}^{2}\left\|(J+A)^{-1}\right\|\right)^{3}=\frac{1}{3}\left\|(J+A)^{-1}\right\|^{3} \operatorname{Tr} B^{3} .
\end{aligned}
$$

Finally,

$$
|\operatorname{Tr} M-\operatorname{Tr} J B|=\left|\operatorname{Tr}\left(B^{1 / 2}\left((J+A)^{-1}-J\right) B^{1 / 2}\right)\right|=\left|\operatorname{Tr}\left(B J A(J+A)^{-1}\right)\right| .
$$

This proves estimate (5.2).

Now, using the notation (3.18), (3.20), and (4.10), we employ Lemma 5.1 to prove Theorem [2.1] and Theorem [2.4] 
5.2. Proof of Theorem 2.1. We shall use the representation (3.21) and Lemma 5.1 Denote $q_{0}=[\mathcal{E} / 2]$. Suppose that $b$ is so large that $\mathcal{E} b+\lambda<2\left(q_{0}+1\right) b$ for all $\lambda \in \Delta$. Then, by (4.12),

$$
\begin{aligned}
\pi^{-1} \operatorname{Tr} & J B(\mathcal{E} b+\lambda) \\
& =\frac{b}{4 \pi^{2}} \sum_{q=0}^{q_{0}}(\mathcal{E} b+\lambda-2 b q)^{-1 / 2} \int_{\mathbb{R}^{3}} V(\mathbf{x}) d \mathbf{x} \\
& =\frac{b^{1 / 2}}{4 \pi^{2}} \sum_{q=0}^{q_{0}}(\mathcal{E}-2 q)^{-1 / 2} \int_{\mathbb{R}^{3}} V(\mathbf{x}) d \mathbf{x}+O(1), \quad b \rightarrow+\infty,
\end{aligned}
$$

uniformly over $\lambda \in \Delta$. Next, by (4.11),

$$
\sup _{\lambda \in \Delta}\|T(\mathcal{E} b+\lambda+i 0)\|=O\left(b^{-1 / 2}\right), \quad b \rightarrow \infty .
$$

It follows that for all sufficiently large $b$ we have

$$
\sup _{\lambda \in \Delta}\|A(\mathcal{E} b+\lambda+i 0)\|<1 / 2
$$

and therefore $\operatorname{ind}(J+A(\mathcal{E} b+\lambda), J)=0, \lambda \in \Delta$.

We estimate the error terms given by the right-hand side of (5.2). By (5.5), for all sufficiently large $b$ we have $\sup _{\lambda \in \Delta}\left\|(J+A(\mathcal{E} b+\lambda))^{-1}\right\| \leq 2$. Using this, relations (5.3) and (5.4), and Lemma 4.2, we obtain

$$
\begin{gathered}
\left\|(J+A)^{-1}\right\|^{3} \operatorname{Tr} B^{3} \leq 2\|B\|^{2} \operatorname{Tr} B=O\left(b^{-1}\right) O\left(b^{1 / 2}\right)=O\left(b^{-1 / 2}\right), \quad b \rightarrow \infty, \\
\left|\operatorname{Tr}\left(B J A(J+A)^{-1}\right)\right| \leq \operatorname{Tr} B\|A\|\left\|(J+A)^{-1}\right\|=O\left(b^{1 / 2}\right) O\left(b^{-1 / 2}\right)=O(1), \quad b \rightarrow \infty,
\end{gathered}
$$

uniformly over $\lambda \in \Delta$. This completes the proof of Theorem 2.1.

\subsection{Proof of Theorem 2.4.}

Lemma 5.2. Assume that (1.5) is true and that the partial derivatives of $\left\langle x_{3}\right\rangle^{m_{3}} V$ with respect to the variables $X_{\perp} \in \mathbb{R}^{2}$ exist and are uniformly bounded on $\mathbb{R}^{3}$. Then $\lim _{b \rightarrow \infty}\left\|\left(1-P_{q_{0}}\right)\left\langle x_{3}\right\rangle^{m_{3}} V P_{q_{0}}\right\|=0$, where $P_{q}=p_{q} \otimes I$ in $L^{2}\left(\mathbb{R}^{3}\right)=L^{2}\left(\mathbb{R}^{2}, d X_{\perp}\right) \otimes$ $L^{2}\left(\mathbb{R}, d x_{3}\right)$.

The proof of Lemma 5.2 can be found in Subsection 9.2.

5.3.1. Proof of Theorem 2.4 We shall use the representation (3.21) and Lemma 5.1

1. First, observe that for any $\lambda<0$ the operator $T_{q_{0}}\left(2 q_{0} b+\lambda\right)$ is selfadjoint and nonnegative and belongs to the trace class. We prove that

$$
\begin{gathered}
\operatorname{ind}\left(J+T_{q_{0}}\left(2 q_{0} b+\lambda\right), J\right)=0, \quad \lambda \in \Delta, \\
\sup _{\lambda \in \Delta}\left\|\left(J+T_{q_{0}}\left(2 q_{0} b+\lambda\right)\right)^{-1}\right\| \leq C,
\end{gathered}
$$

where $C$ does not depend on $b>0$. Consider the operator $I \otimes \chi_{0}+V$ in $L^{2}\left(\mathbb{R}^{3}\right)=$ $L^{2}\left(\mathbb{R}^{2}, d X_{\perp}\right) \otimes L^{2}\left(\mathbb{R}, d x_{3}\right)$. Our assumption $\Delta \subset(-\infty, \Lambda)$ means that $\sup \Delta<\Lambda=$ $\inf \sigma\left(I \otimes \chi_{0}+V\right)$; therefore, by the Birman-Schwinger principle,

$$
\sup _{\lambda \in \Delta}\left\|\left(I \otimes \chi_{0}-\lambda\right)^{-1 / 2} V\left(I \otimes \chi_{0}-\lambda\right)^{-1 / 2}\right\|<1 .
$$

It follows that

$$
\sup _{\lambda \in \Delta}\left\|\left(p_{q_{0}} \otimes\left(\chi_{0}-\lambda\right)^{-1 / 2}\right) V\left(p_{q_{0}} \otimes\left(\chi_{0}-\lambda\right)^{-1 / 2}\right)\right\|<1,
$$


or equivalently,

$$
\sup _{\lambda \in \Delta}\left\|\left(T_{q_{0}}\left(2 q_{0} b+\lambda\right)\right)^{1 / 2} J\left(T_{q_{0}}\left(2 q_{0} b+\lambda\right)\right)^{1 / 2}\right\|<1 .
$$

From this, using the formula

$$
\left(J+T_{q_{0}}\right)^{-1}=J-J T_{q_{0}}^{1 / 2}\left(I+T_{q_{0}}^{1 / 2} J T_{q_{0}}^{1 / 2}\right)^{-1} T_{q_{0}}^{1 / 2} J
$$

and estimate (4.8), we obtain (5.7). Using (3.9) and (5.8), we get (5.6).

2. Next, as in (4.11) we have

$$
\begin{aligned}
\sup _{\lambda \in \Delta} \| & A\left(2 b q_{0}+\lambda\right)-T_{q_{0}}\left(2 b q_{0}+\lambda\right) \| \\
& \leq C_{0} \sup _{\lambda \in \Delta}\left\|\left\langle x_{3}\right\rangle^{-m_{3} / 2}\left(\sum_{q \neq q_{0}} p_{q} \otimes r\left(2 b\left(q_{0}-q\right)+\lambda+i 0\right)\right)\left\langle x_{3}\right\rangle^{-m_{3} / 2}\right\| \\
& \leq C_{0} C\left(m_{3}\right) \sup \left\{\left|2 b\left(q_{0}-q\right)+\lambda\right|^{-1 / 2} \mid q \in \mathbb{Z}_{+}, q \neq q_{0}, \lambda \in \Delta\right\} \\
& =O\left(b^{-1 / 2}\right), \quad b \rightarrow \infty .
\end{aligned}
$$

From this and (5.6), by the stability of the index, it follows that

$$
\operatorname{ind}\left(J+A\left(2 b q_{0}+\lambda\right), J\right)=0, \quad \lambda \in \Delta,
$$

for all sufficiently large $b$. Also, (5.7) and (5.9) imply

$$
\sup _{\lambda \in \Delta}\left\|\left(J+A\left(2 b q_{0}+\lambda\right)\right)^{-1}\right\|=O(1), \quad b \rightarrow \infty .
$$

3. We apply Lemma 5.1. For the leading term we have formula (4.12), which gives the limiting expression in (2.5). Now, we check that the remainder terms given by the right-hand side of (5.2) are $o\left(b^{1 / 2}\right)$. Consider the term $\left\|(J+A)^{-1}\right\|^{3} \operatorname{Tr} B^{3}$. For $\lambda<0$ we have

$$
B\left(2 b q_{0}+\lambda\right)=\sum_{q=0}^{q_{0}-1} \operatorname{Im} T_{q}\left(2 b q_{0}+\lambda\right) .
$$

Consequently, by (5.3) and (4.11),

$$
\sup _{\lambda \in \Delta} \operatorname{Tr} B^{3}\left(2 b q_{0}+\lambda\right)=O\left(b^{-\frac{1}{2}}\right) .
$$

Combining this with (5.11), we get the required bound for $\left\|(J+A)^{-1}\right\|^{3} \operatorname{Tr} B^{3}$.

4. Finally, consider the term $\operatorname{Tr}\left(B J A(J+A)^{-1}\right)$. We prove that

$$
\sup _{\lambda \in \Delta}\left\|B\left(2 q_{0} b+\lambda\right) J A\left(2 q_{0} b+\lambda\right)\right\|_{S_{1}}=o\left(b^{1 / 2}\right), \quad b \rightarrow \infty .
$$

By (5.9) and (4.12), it suffices to prove that

$$
\sup _{\lambda \in \Delta}\left\|B\left(2 q_{0} b+\lambda\right) J T_{q_{0}}\left(2 q_{0} b+\lambda\right)\right\|_{S_{1}}=o\left(b^{1 / 2}\right), \quad b \rightarrow \infty .
$$

By (5.12), it suffices to consider $\operatorname{Im}\left(T_{q}\left(2 q_{0} b+\lambda\right)\right) J T_{q_{0}}\left(2 q_{0} b+\lambda\right)$ for any fixed $q<q_{0}$. Using the notation $M(\mathbf{x})=|V(\mathbf{x})|\left\langle X_{\perp}\right\rangle^{m_{\perp}}\left\langle x_{3}\right\rangle^{m_{3}}$ and $V_{\perp}\left(X_{\perp}, x_{3}\right):=\left\langle x_{3}\right\rangle^{m_{3}} V\left(X_{\perp}, x_{3}\right)$, we obtain

$$
\begin{aligned}
& \operatorname{Im}\left(T_{q}\left(2 q_{0} b+\lambda\right)\right) J T_{q_{0}}\left(2 q_{0} b+\lambda\right) \\
& \quad=M^{1 / 2}\left(\left\langle X_{\perp}\right\rangle^{-m_{\perp} / 2} p_{q} \otimes \operatorname{Im} t\left(2 b\left(q_{0}-q\right)+\lambda\right)\right) P_{q} V_{\perp} P_{q_{0}}\left(p_{q_{0}}\left\langle X_{\perp}\right\rangle^{-m_{\perp}} \otimes t(\lambda)\right) M^{1 / 2} .
\end{aligned}
$$

By Lemma 5.2, as $b$ tends to infinity, we have $\left\|P_{q} V_{\perp} P_{q_{0}}\right\|=o(1)$. Next, (4.3) and (4.7) show that

$$
\begin{gathered}
\left\|\left\langle X_{\perp}\right\rangle^{m_{\perp} / 2} p_{q} \otimes \operatorname{Im} t\left(2 b\left(q_{0}-q\right)+\lambda\right)\right\|_{S_{2}} \leq C b^{1 / 2}\left|2 b\left(q_{0}-q\right)-\lambda\right|^{-1 / 2}=O(1), \quad b \rightarrow \infty \\
\left\|p_{q_{0}}\left\langle X_{\perp}\right\rangle^{m_{\perp} / 2} \otimes t(\lambda)\right\|_{S_{2}}=O\left(b^{1 / 2}\right), \quad b \rightarrow \infty
\end{gathered}
$$


uniformly over $\lambda \in \Delta$. Combining the above bounds, we get (5.14).

\section{§6. Properties of $\xi\left(\lambda ; \chi\left(X_{\perp}\right), \chi_{0}\right)$}

Here we prepare some auxiliary statements for the proof of Theorem [2.4. These statements pertain to the $\operatorname{SSF} \xi\left(\lambda ; \chi\left(X_{\perp}\right), \chi_{0}\right)$. We shall use the representation (3.15) for $\xi\left(\lambda ; \chi\left(X_{\perp}\right), \chi_{0}\right)$. With the notation

$$
\begin{aligned}
& \tau\left(X_{\perp}, \lambda\right)=\left|V\left(X_{\perp}, \cdot\right)\right|^{1 / 2}\left(\chi_{0}-\lambda-i 0\right)^{-1}\left|V\left(X_{\perp}, \cdot\right)\right|^{1 / 2}, \\
\iota\left(X_{\perp}\right)= & \operatorname{sgn} V\left(X_{\perp}, \cdot\right), \quad \alpha\left(X_{\perp}, \lambda\right)=\operatorname{Re} \tau\left(X_{\perp}, \lambda\right), \quad \beta\left(X_{\perp}, \lambda\right)=\operatorname{Im} \tau\left(X_{\perp}, \lambda\right),
\end{aligned}
$$

this representation reads

$$
\begin{aligned}
& \xi(\lambda ; \chi \\
& \quad=\int_{-\infty}^{\infty} \operatorname{ind}\left(\iota\left(X_{\perp}\right)+\alpha\left(X_{\perp}, \lambda\right)+t \beta\left(X_{\perp}, \lambda\right), \iota\left(X_{\perp}\right)\right) d \mu(t) \quad \text { for a.e. } \lambda \in \mathbb{R} .
\end{aligned}
$$

Along with the operator $\chi\left(X_{\perp}\right)$, we consider its modification: for $\eta \in \mathbb{R},|\eta| \neq 1$, let

$$
\chi\left(X_{\perp}, \eta\right)=\chi_{0}+\left|V\left(X_{\perp}, \cdot\right)\right|\left(\iota\left(X_{\perp}\right)-\eta\right)^{-1} .
$$

By (3.15), for this operator we have

$$
\xi\left(\lambda ; \chi\left(X_{\perp}, \eta\right), \chi_{0}\right)=\int_{-\infty}^{\infty} \Xi_{\lambda}\left(\eta, X_{\perp}, t\right) d \mu(t) \quad \text { for a.e. } \lambda \in \mathbb{R},
$$

where

$$
\Xi_{\lambda}\left(\eta, X_{\perp}, t\right):=\operatorname{ind}\left(\iota\left(X_{\perp}\right)+\alpha\left(X_{\perp}, \lambda\right)+t \beta\left(X_{\perp}, \lambda\right)-\eta, \iota\left(X_{\perp}\right)-\eta\right), \quad|\eta| \neq 1 .
$$

We shall need some continuity and measurability properties of $\Xi_{\lambda}\left(\eta, X_{\perp}, t\right)$.

Lemma 6.1. (i) The set

$$
\Omega=\left\{\left(\lambda, \eta, X_{\perp}, t\right)\left|\eta \notin \sigma\left(\iota\left(X_{\perp}\right)+\alpha\left(X_{\perp}, \lambda\right)+t \beta\left(X_{\perp}, \lambda\right)\right), \lambda \neq 0,\right| \eta \mid \neq 1\right\}
$$

is open in $\mathbb{R} \times \mathbb{R} \times \mathbb{R}^{2} \times \mathbb{R}$, and the function $\Xi_{\lambda}\left(\eta, X_{\perp}, t\right)$ is constant on the connected components of this set.

(ii) The function $\Xi_{\lambda}\left(\eta, X_{\perp}, t\right)$ is lower semicontinuous on the set

$$
\left\{\left(\lambda, \eta, X_{\perp}, t\right)|\lambda \neq 0,| \eta \mid \neq 1, X_{\perp} \in \mathbb{R}^{2}, t \in \mathbb{R}\right\} .
$$

Proof. 1. First, consider the difference of resolvents

$$
\left(\iota\left(X_{\perp}\right)+\alpha\left(X_{\perp}, \lambda\right)+t \beta\left(X_{\perp}, \lambda\right)-z\right)^{-1}-\left(\iota\left(X_{\perp}\right)-z\right)^{-1}
$$

for a fixed $z$ away from the spectra of $\iota\left(X_{\perp}\right)$ and $\iota\left(X_{\perp}\right)+\alpha\left(X_{\perp}, \lambda\right)+t \beta\left(X_{\perp}, \lambda\right)$. We prove that this difference depends continuously on $\lambda, X_{\perp}$, and $t$ in the trace norm. Here the only nontrivial issue is the continuous dependence on $X_{\perp}$ (observe that $\iota\left(X_{\perp}\right)$ is not continuous in $X_{\perp}$ even in the operator norm). In order to prove the continuous dependence on $X_{\perp}$, first we observe that the operators $\alpha\left(X_{\perp}, \lambda\right)+t \beta\left(X_{\perp}, \lambda\right), \iota\left(X_{\perp}\right)\left(\alpha\left(X_{\perp}, \lambda\right)+t \beta\left(X_{\perp}, \lambda\right)\right)$, $\left(\alpha\left(X_{\perp}, \lambda\right)+t \beta\left(X_{\perp}, \lambda\right)\right) \iota\left(X_{\perp}\right)$, and $\iota\left(X_{\perp}\right)\left(\alpha\left(X_{\perp}, \lambda\right)+t \beta\left(X_{\perp}, \lambda\right)\right) \iota\left(X_{\perp}\right)$ depend continuously on $X_{\perp}$ in the trace norm. Using the identity

$(\iota+\alpha+t \beta-z)^{-1}-(\iota-z)^{-1}=-\left(I+(\iota-z)^{-1}(\alpha+t \beta)\right)^{-1}(\iota-z)^{-1}(\alpha+t \beta)(\iota-z)^{-1}$, and expanding $(\iota-z)^{-1}$ in a norm convergent series, we obtain the desired statement.

2. From the result of the preceding step, we deduce that the eigenvalues of $\iota\left(X_{\perp}\right)+$ $\alpha\left(X_{\perp}, \lambda\right)+t \beta\left(X_{\perp}, \lambda\right)$ depend continuously on $\lambda, X_{\perp}$, and $t$. This proves that $\Omega$ is open. Since $\alpha\left(X_{\perp}, \lambda\right)+t \beta\left(X_{\perp}, \lambda\right)$ is of trace class, we have

$$
\begin{gathered}
\Xi_{\lambda}\left(\eta, X_{\perp}, t\right)=\operatorname{index}\left(E_{\iota\left(X_{\perp}\right)+\alpha\left(X_{\perp}, \lambda\right)+t \beta\left(X_{\perp}, \lambda\right)}((-\infty, \eta)), E_{\iota\left(X_{\perp}\right)}((-\infty, \eta))\right) \\
=\operatorname{Tr}\left(E_{\iota\left(X_{\perp}\right)+\alpha\left(X_{\perp}, \lambda\right)+t \beta\left(X_{\perp}, \lambda\right)}((-\infty, \eta))-E_{\iota\left(X_{\perp}\right)}((-\infty, \eta))\right) .
\end{gathered}
$$


Representing the above spectral projections as Riesz integrals, and using the result of the first step of the proof, we see that $\Xi_{\lambda}\left(\eta, X_{\perp}, t\right)$ is continuous on $\Omega$. Since $\Xi_{\lambda}\left(\eta, X_{\perp}, t\right)$ is integer-valued, it is constant on the connected components of $\Omega$. Precisely the same argument shows also that $\Xi_{\lambda}\left(\eta, X_{\perp}, t\right)$ is lower semicontinuous on the entire range of its variables.

Recall that the lower semicontinuous functions are measurable. Thus, Lemma 6.1 ensures that $\Xi_{\lambda}\left(\eta, X_{\perp}, t\right)$ is measurable with respect to any combination of its variables. We shall need to integrate $\Xi_{\lambda}\left(\eta, X_{\perp}, t\right)$ with respect to some of its variables; integrability is provided by the following statement.

Lemma 6.2. (i) For any $p \in(0,1)$, we have the bound

$$
\sup _{|\eta| \leq 1 / 2}\left|\Xi_{\lambda}\left(\eta, X_{\perp}, t\right)\right| \leq C\left(1+\lambda_{+}^{1 / 4}\right)|\lambda|^{-1 / 2}\left\langle X_{\perp}\right\rangle^{-m_{\perp}}+C|\lambda|^{-p / 2} t^{p}\left\langle X_{\perp}\right\rangle^{-p m_{\perp}} .
$$

(ii) For any $t \in \mathbb{R}$ and any $\Delta \subset \mathbb{R} \backslash\{0\}$, we have

$$
\sup _{\lambda \in \Delta} \int_{-\infty}^{\infty} d \eta \int_{\mathbb{R}^{2}} d X_{\perp}\left|\Xi_{\lambda}\left(\eta, X_{\perp}, t\right)\right|<\infty .
$$

Proof. (i) Recall that $\tau\left(X_{\perp}, \lambda\right)$ is a trace class operator satisfying (4.4), and $\beta\left(X_{\perp}, \lambda\right)$ has rank two and satisfies (4.5). Thus, by estimate (1.5) on $V$, we have

$$
\begin{aligned}
& \left\|\alpha\left(X_{\perp}, \lambda\right)\right\|_{S_{1}} \leq \frac{C}{\sqrt{|\lambda|}}\left(1+\lambda_{+}^{1 / 4}\right)\left\langle X_{\perp}\right\rangle^{-m_{\perp}}, \\
& \left\|\beta\left(X_{\perp}, \lambda\right)\right\|_{S_{p}}^{p} \leq C|\lambda|^{-p / 2}\left\langle X_{\perp}\right\rangle^{-p m_{\perp}} .
\end{aligned}
$$

Using these bounds and arguing as in (3.16) and (3.17), for any $p \in(0,1)$ we obtain

$$
\begin{aligned}
\sup _{|\eta| \leq 1 / 2}\left|\Xi_{\lambda}\left(\eta, X_{\perp}, t\right)\right| & \leq n_{*}\left(1 / 4, \alpha\left(X_{\perp}, \lambda\right)+t \beta\left(X_{\perp}, \lambda\right)\right) \\
& \leq n_{*}\left(1 / 8, \alpha\left(X_{\perp}, \lambda\right)\right)+n_{*}\left(1 / 8, t \beta\left(X_{\perp}, \lambda\right)\right) \\
& \leq\left\|8 \alpha\left(X_{\perp}, \lambda\right)\right\|_{S_{1}}+\left\|8 t \beta\left(X_{\perp}, \lambda\right)\right\|_{S_{p}}^{p} \\
& \leq C\left(1+\lambda_{+}^{1 / 4}\right)|\lambda|^{-1 / 2}\left\langle X_{\perp}\right\rangle^{-m_{\perp}}+C|\lambda|^{-p / 2} t^{p}\left\langle X_{\perp}\right\rangle^{-p m_{\perp}} .
\end{aligned}
$$

(ii) First, observe that, by property (a) of ind,

$$
\Xi_{\lambda}\left(\eta, X_{\perp}, t\right)=-\lim _{\epsilon \rightarrow+0} \xi\left(\eta-\epsilon ; \iota\left(X_{\perp}\right)+\alpha\left(X_{\perp}, \lambda\right)+t \beta\left(X_{\perp}, \lambda\right), \iota\left(X_{\perp}\right)\right) .
$$

Using this fact and KreĬn's bound for the SSF (see, e.g., [31, Theorem 8.2.1]), we obtain

$$
\begin{gathered}
\int_{-\infty}^{\infty} d \eta \int_{\mathbb{R}^{2}} d X_{\perp}\left|\xi\left(\eta ; \iota\left(X_{\perp}\right)+\alpha\left(X_{\perp}, \lambda\right)+t \beta\left(X_{\perp}, \lambda\right), \iota\left(X_{\perp}\right)\right)\right| \\
\quad \leq \int_{\mathbb{R}^{2}}\left\|\alpha\left(X_{\perp}, \lambda\right)+t \beta\left(X_{\perp}, \lambda\right)\right\|_{S_{1}} d X_{\perp}<\infty
\end{gathered}
$$

This completes the proof.

Lemma 6.3. (i) For each $\eta \in\left[-\frac{1}{2}, \frac{1}{2}\right]$ and $X_{\perp} \in \mathbb{R}^{2}$, the function $\int_{-\infty}^{\infty} \Xi_{\lambda}\left(\eta, X_{\perp}, t\right) d \mu(t)$ is continuous for $\lambda>0$ and right continuous for $\lambda<0$.

(ii) The function $\int_{\mathbb{R}^{2}} d X_{\perp} \int_{-\infty}^{\infty} d \mu(t) \Xi_{\lambda}\left(\eta, X_{\perp}, t\right)$ is continuous for $\lambda>0$ and $\eta \in$ $[-1 / 2,1 / 2]$.

Proof. (i) First, we prove continuity for $\lambda>0$. We shall use the dominated convergence theorem; Lemma 6.2(i) gives an integrable bound, and Lemma 6.1(i) provides the continuity of the integrand away from the set $S:=\left\{t \mid \eta \in \sigma\left(\iota\left(X_{\perp}\right)+\alpha\left(X_{\perp}, \lambda\right)+t \beta\left(X_{\perp}, \lambda\right)\right)\right\}$. 
We need only to prove that this set has zero measure in $\mathbb{R}$. By the analytic Fredholm alternative, the relation $t \in S$ is fulfilled either for countably many $t$ or for all $t \in \mathbb{C}$. We show that the latter is impossible. Indeed, if $i \in S$, then $-1 \in$ $\sigma\left(\left(\iota\left(X_{\perp}\right)-\eta\right)^{-1}\left(\alpha\left(X_{\perp}, \lambda\right)+i \beta\left(X_{\perp}, \lambda\right)\right)\right)$, and Agmon's classical bootstrap argument shows that $\lambda>0$ is an eigenvalue of the one-dimensional Schrödinger operator $\chi\left(X_{\perp}, \eta\right)$, which is impossible. This proves continuity in the domain $\lambda>0$.

The lower semicontinuity of $\Xi_{\lambda}\left(\eta, X_{\perp}, t\right)$ is inherited by its integral over $t$. Also, the function $\Xi_{\lambda}\left(\eta, X_{\perp}, t\right)$ (and, therefore, its integral) is monotone nonincreasing for $\lambda<0$. It follows that the integral of $\Xi_{\lambda}\left(\eta, X_{\perp}, t\right)$ is right continuous for $\lambda<0$.

Statement (ii) can be proved by following the same pattern.

Proof of Proposition [2.2. By Lemma 6.3]i), with our choice of $\xi\left(\lambda ; \chi\left(X_{\perp}\right), \chi_{0}\right)$ (continuous in $\lambda>0$ and right continuous in $\lambda<0$ ), identity (6.3) is true for all $\lambda \neq 0$. Then the integrability of $\xi\left(\lambda ; \chi\left(X_{\perp}\right), \chi_{0}\right)$ is ensured by (6.6) with $2 / m_{\perp}<p<1$, and continuity for $\lambda>0$ is ensured by Lemma 6.3. In view of (1.3), the last statement is obvious.

\section{$\S 7$. Asymptotic TRACE FORMUlas}

In this section, we establish the key limit relation used in the proof of Theorem 2.3 Let $T_{q}(E)$ be as above (see $(4.10)$ ); we denote

$$
A_{q}=\operatorname{Re} T_{q}(2 q b+\lambda), \quad B_{q}=\operatorname{Im} T_{q}(2 q b+\lambda), \quad J=\operatorname{sgn} V .
$$

Moreover, we use the notation introduced in (6.1)- 6.2 .

Proposition 7.1. Suppose (1.5) is fulfilled, and let $\Delta$ be a compact interval in $\mathbb{R} \backslash\{0\}$. Let $r=\left(r_{1}, \ldots, r_{p}\right) \in\{0,1\}^{p}$ with $p \geq 1$. Then for every $t \in \mathbb{R}$ we have

$$
\begin{gathered}
\lim _{b \rightarrow \infty} b^{-1} \operatorname{Tr}\left(J^{r_{1}}\left(A_{q_{0}}+t B_{q_{0}}\right) \cdots J^{r_{p}}\left(A_{q_{0}}+t B_{q_{0}}\right)\right) \\
=\frac{1}{2 \pi} \int_{\mathbb{R}^{2}} \operatorname{Tr}\left(\iota\left(X_{\perp}\right)^{r_{1}}\left(\alpha\left(X_{\perp}, \lambda\right)+t \beta\left(X_{\perp}, \lambda\right)\right) \cdots\right. \\
\left.\iota\left(X_{\perp}\right)^{r_{p}}\left(\alpha\left(X_{\perp}, \lambda\right)+t \beta\left(X_{\perp}, \lambda\right)\right)\right) d X_{\perp},
\end{gathered}
$$

where the convergence is uniform in $\lambda \in \Delta$.

Proof. We have

$$
\begin{aligned}
\operatorname{Tr}\left(J^{r_{1}}\left(A_{q_{0}}+t B_{q_{0}}\right) \cdots J^{r_{p}}\left(A_{q_{0}}+t B_{q_{0}}\right)\right) \\
=\int_{\mathbb{R}^{2 p}} \int_{\mathbb{R}^{p}} \prod_{j=1}^{p} W_{j}\left(X_{\perp, j}, x_{3, j}\right) \\
\quad \times \prod_{j=1}^{p} K_{q_{0}, b}\left(X_{\perp, j}, X_{\perp, j+1}\right) \mathcal{R}_{\lambda, t}\left(x_{3, j}-x_{3, j+1}\right) \prod_{j=1}^{p} d X_{\perp, j} d x_{3, j},
\end{aligned}
$$

where $W_{j}=(\operatorname{sgn} V)^{r_{j}}|V|$,

$$
\mathcal{R}_{\lambda, t}(x):=\left\{\begin{array}{ll}
-\frac{\sin (\sqrt{\lambda}|x|)}{2 \sqrt{\lambda}}+t \frac{\cos (\sqrt{\lambda} x)}{2 \sqrt{\lambda}} & \text { if } \lambda>0, \\
\frac{e^{-\sqrt{-\lambda}|x|}}{2 \sqrt{-\lambda}} & \text { if } \lambda<0,
\end{array} \quad t \in \mathbb{R}, \quad x \in \mathbb{R}\right.
$$

and the notation $\prod_{j=1}^{\prime p}$ means that, in the product of $p$ factors, the variables $X_{\perp, p+1}$ and $x_{3, p+1}$ should be set equal (respectively) to $X_{\perp, 1}$ and $x_{3,1}$. For $p=1$ the claimed asymptotic is an identity because $K_{q, b}\left(X_{\perp}, X_{\perp}\right)=\frac{b}{2 \pi}$. For $p \geq 2$, we change the variables: 


$$
X_{\perp, 1}=X_{\perp, 1}^{\prime}, \quad X_{\perp, j}=X_{\perp, 1}^{\prime}+b^{-1 / 2} X_{\perp, j}^{\prime}, \quad j=2, \ldots, p .
$$

Thus, we obtain

$$
\begin{aligned}
\operatorname{Tr}\left(J^{r_{1}}\left(A_{q_{0}}+t B_{q_{0}}\right) \cdots J^{r_{p}}\left(A_{q_{0}}+t B_{q_{0}}\right)\right) & \\
=b \int_{\mathbb{R}^{2 p}} \int_{\mathbb{R}^{p}} & W_{1}\left(X_{\perp, 1}^{\prime}, x_{3,1}\right) \prod_{j=2}^{p} W_{j}\left(X_{\perp, 1}^{\prime}+b^{-1 / 2} X_{\perp, j}^{\prime}, x_{3, j}\right) K_{q_{0}, 1}\left(0, X_{\perp, 2}^{\prime}\right) \\
& \times \prod_{j=2}^{p-1} K_{q_{0}, 1}\left(X_{\perp, j}^{\prime}, X_{\perp, j+1}^{\prime}\right) K_{q_{0}, 1}\left(X_{\perp, p}^{\prime}, 0\right) \\
& \times \prod_{j=1}^{p} \mathcal{R}_{\lambda, t}\left(x_{3, j}-x_{3, j+1}\right) \prod_{j=1}^{p} d X_{\perp, j}^{\prime} d x_{3, j} .
\end{aligned}
$$

Here and in the sequel, for $p=2$ the product $\prod_{j=2}^{p-1} K_{q_{0}, b}\left(X_{\perp, j}, X_{\perp, j+1}\right)$ should be set equal to one. The modulus of the integrand on the right-hand side of (7.4) is upper bounded by the $L^{1}\left(\mathbb{R}^{3 p}\right)$-function

$$
\left(\frac{1+|t|}{2 \sqrt{|\lambda|}}\right)^{p}\left\langle X_{\perp, 1}^{\prime}\right\rangle^{-m_{\perp}} \mathcal{P}\left(X_{\perp, 2}^{\prime}, \ldots, X_{\perp, p}^{\prime}\right) e^{-g \sum_{j=2}^{p}\left|X_{\perp, j}^{\prime}\right|^{2}} \prod_{j=1}^{p}\left\langle x_{3, j}\right\rangle^{-m_{3}},
$$

where $\mathcal{P}$ is a polynomial and $g$ is a positive constant. Moreover, for each $\left(X_{\perp, 1}{ }^{\prime}, x_{3,1}, \ldots\right.$, $\left.X_{\perp, p}^{\prime}, x_{3, p}\right)$ we have

$$
\lim _{b \rightarrow \infty} W_{1}\left(X_{\perp, 1}^{\prime}, x_{3,1}\right) \prod_{j=2}^{p} W_{j}\left(X_{\perp, 1}^{\prime}+b^{-1 / 2} X_{\perp, j}^{\prime}, x_{3, j}\right)=\prod_{j=1}^{p} W_{j}\left(X_{\perp, 1}^{\prime}, x_{3, j}\right) .
$$

Applying the dominated convergence theorem, we see that (7.4) entails

$$
\begin{aligned}
\lim _{b \rightarrow \infty} b^{-1} \operatorname{Tr}\left(J^{r_{1}}\left(A_{q_{0}}+t B_{q_{0}}\right) \cdots J^{r_{p}}\left(A_{q_{0}}+t B_{q_{0}}\right)\right) \\
=\int_{\mathbb{R}^{2}} \int_{\mathbb{R}^{p}} \prod_{j=1}^{p} W_{j}\left(X_{\perp, 1}, x_{3, j}\right) \prod_{j=1}^{p} \mathcal{R}_{\lambda, t}\left(x_{3, j}-x_{3, j+1}\right) d X_{\perp, 1} \prod_{j=1}^{p} d x_{3, j} \\
\quad \times \int_{\mathbb{R}^{2(p-1)}} K_{q_{0}, 1}\left(0, X_{\perp, 2}\right) \prod_{j=2}^{p-1} K_{q_{0}, 1}\left(X_{\perp, j}, X_{\perp, j+1}\right) K_{q_{0}, 1}\left(X_{\perp, p}, 0\right) \prod_{j=2}^{p} d X_{\perp, j} \\
=\int_{\mathbb{R}^{2}} \operatorname{Tr}\left(\iota\left(X_{\perp, 1}\right)^{r_{1}}\left(\alpha\left(X_{\perp, 1}, \lambda\right)+t \beta\left(X_{\perp, 1}, \lambda\right)\right)\right. \\
\left.\quad \cdots \iota\left(X_{\perp, 1}\right)^{r_{p}}\left(\alpha\left(X_{\perp, 1}, \lambda\right)+t \beta\left(X_{\perp, 1}, \lambda\right)\right)\right) d X_{\perp, 1} \\
\quad \times \int_{\mathbb{R}^{2(p-1)}} K_{q_{0}, 1}\left(0, X_{\perp, 2}\right) \prod_{j=2}^{p-1} K_{q_{0}, 1}\left(X_{\perp, j}, X_{\perp, j+1}\right) K_{q_{0}, 1}\left(X_{\perp, p}, 0\right) \prod_{j=2}^{p} d X_{\perp, j},
\end{aligned}
$$

uniformly in $\lambda \in \Delta$. In order to conclude that (7.5) is equivalent to (7.2), it remains to note that

$$
\begin{gathered}
\int_{\mathbb{R}^{2(p-1)}} K_{q_{0}, 1}\left(0, X_{\perp, 2}\right) \prod_{j=2}^{p-1} K_{q_{0}, 1}\left(X_{\perp, j}, X_{\perp, j+1}\right) K_{q_{0}, 1}\left(X_{\perp, p}, 0\right) \prod_{j=2}^{p} d X_{\perp, j} \\
=K_{q_{0}, 1}(0,0)=\frac{1}{2 \pi} . \quad \square
\end{gathered}
$$


Corollary 7.2. For each polynomial $\phi$ and $t \in \mathbb{R}$, we have

$$
\begin{aligned}
\lim _{b \rightarrow \infty} & b^{-1} \operatorname{Tr}\left(\phi\left(J+A_{q_{0}}+t B_{q_{0}}\right)-\phi(J)\right) \\
& =\frac{1}{2 \pi} \int_{\mathbb{R}^{2}} d X_{\perp} \operatorname{Tr}\left(\phi\left(\iota\left(X_{\perp}\right)+\alpha\left(X_{\perp}, \lambda\right)+t \beta\left(X_{\perp}, \lambda\right)\right)-\phi\left(\iota\left(X_{\perp}\right)\right)\right),
\end{aligned}
$$

where the convergence is uniform in $\lambda$ on the compact subsets of $\mathbb{R} \backslash\{0\}$.

Proof. By the linearity of the trace and the integral, it suffices to prove the lemma for $\phi(x)=x^{k}, k \in \mathbb{Z}_{+}$. Using the cyclicity of the trace and the identity $J^{2}=I$, we find that $\operatorname{Tr}\left(\left(J+A_{q_{0}}+t B_{q_{0}}\right)^{k}-J^{k}\right)$ can be written as the trace of a linear combination of operators of the form $J^{r_{1}}\left(A_{q_{0}}+t B_{q_{0}}\right) \cdots J^{r_{p}}\left(A_{q_{0}}+t B_{q_{0}}\right)$ with $r=\left(r_{1}, \ldots, r_{p}\right) \in\{0,1\}^{p}$ and $1 \leq p \leq k$. Similarly, $\operatorname{Tr}\left(\left(\iota\left(X_{\perp}\right)+\alpha\left(X_{\perp}, \lambda\right)+t \beta\left(X_{\perp}, \lambda\right)\right)^{k}-\iota\left(X_{\perp}\right)^{k}\right)$ with fixed $X_{\perp} \in \mathbb{R}^{2}$ can be written as the trace of a linear combination of operators of the form

$$
\iota\left(X_{\perp}\right)^{r_{1}}\left(\alpha\left(X_{\perp}, \lambda\right)+t \beta\left(X_{\perp}, \lambda\right)\right) \cdots \iota\left(X_{\perp}\right)^{r_{p}}\left(\alpha\left(X_{\perp}, \lambda\right)+t \beta\left(X_{\perp}, \lambda\right)\right),
$$

which involves the same coefficients as in the case of $\operatorname{Tr}\left(\left(J+A_{q_{0}}+t B_{q_{0}}\right)^{k}-J^{k}\right)$. Using the linearity of the trace and of the integral once again, and applying Proposition 7.1 we deduce:

$$
\begin{aligned}
\lim _{b \rightarrow \infty} & b^{-1} \operatorname{Tr}\left(\left(J+A_{q_{0}}+t B_{q_{0}}\right)^{k}-J^{k}\right) \\
\quad= & \frac{1}{2 \pi} \int_{\mathbb{R}^{2}} \operatorname{Tr}\left(\left(\iota\left(X_{\perp}\right)+\alpha\left(X_{\perp}, \lambda\right)+t \beta\left(X_{\perp}, \lambda\right)\right)^{k}-\iota\left(X_{\perp}\right)^{k}\right) d X_{\perp} .
\end{aligned}
$$

This completes the proof of Corollary 7.2

\section{§8. SSF ASYMptotics of order $b$ : PRoof of Theorem 2.3}

In this section we use the above results and the notation (3.18), (3.20), (4.10), and (7.1) to prove Theorem 2.3.

Lemma 8.1. For each $q \in \mathbb{Z}_{+}$and $p>2 / m_{\perp}$, we have

$$
\left\|p_{q}\left\langle X_{\perp}\right\rangle^{-m_{\perp}} p_{q}\right\|_{S_{p}}^{p}=O(b), \quad b \rightarrow \infty .
$$

The proof of the lemma can be found in Subsection 9.4.

Fix $q_{0} \in \mathbb{Z}_{+}$. All "constants" $C$ appearing in the proof may depend on $V$ and $q_{0}$.

Lemma 8.2. Under the assumption (1.5), for each compact interval $\Delta$ in $\mathbb{R} \backslash\{0\}$ the following statements are valid.

(i) For all $b>0$ and all $q \in \mathbb{Z}_{+}$, we have $T_{q}\left(2 b q_{0}+\lambda\right) \in S_{1}$, and

$$
\sup _{\lambda \in \Delta}\left\|T_{q}\left(2 b q_{0}+\lambda\right)\right\|_{S_{1}}=O\left(b^{3 / 4}\right), \quad b \rightarrow \infty, \quad \text { if } \quad q \neq q_{0},
$$

and

$$
\sup _{\lambda \in \Delta}\left\|T_{q_{0}}\left(2 b q_{0}+\lambda\right)\right\|_{S_{1}}=O(b), \quad b \rightarrow \infty .
$$

(ii) For every $p>2 / m_{\perp}$ and $\lambda \neq 0$, we have $B_{q_{0}}\left(2 b q_{0}+\lambda\right) \in S_{p}$, and

$$
\sup _{\lambda \in \Delta}\left\|B_{q_{0}}\left(2 b q_{0}+\lambda\right)\right\|_{S_{p}}^{p}=O(b), \quad b \rightarrow \infty .
$$

Proof. Note that, by (1.5), the proof reduces to the case where $V(x)=\left\langle X_{\perp}\right\rangle^{-m_{\perp}}\left\langle x_{3}\right\rangle^{-m_{3}}$. We consider this case. We have

$$
T_{q}\left(2 b q_{0}+\lambda\right)=\left\langle X_{\perp}\right\rangle^{-m_{\perp} / 2} p_{q}\left\langle X_{\perp}\right\rangle^{-m_{\perp} / 2} \otimes t\left(2 b\left(q_{0}-q\right)+\lambda\right) .
$$

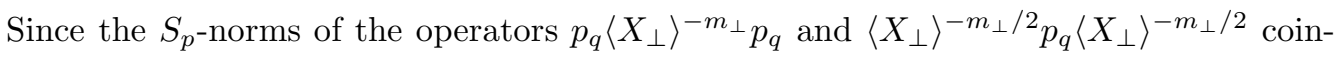
cide, the required statements follow from Lemma 8.1 estimates (4.4) and (4.5), and the fact that $\operatorname{Im} t(\lambda)$ is of rank two. 
Proof of Theorem 2.3. 1. First, we prove that

$$
\begin{aligned}
\limsup _{b \rightarrow \infty} \sup _{\lambda \in \Delta_{1} \cup \Delta_{2}}\left\{b^{-1} \int_{-\infty}^{\infty} \operatorname{ind}(J+A+t B, J) d \mu(t)\right. \\
\left.-\int_{-\infty}^{\infty} b^{-1} \operatorname{ind}\left(J-\eta+A_{q_{0}}+t B_{q_{0}}, J-\eta\right) d \mu(t)\right\} \leq 0
\end{aligned}
$$

for each $\eta \in(0,1)$. By (3.11) and (3.17),

$$
\begin{aligned}
\int_{-\infty}^{\infty} \operatorname{ind}(J+A+t B, J) d \mu(t) \\
\leq \int_{-\infty}^{\infty} \operatorname{ind}\left(J-\eta+A_{q_{0}}+t B_{q_{0}}, J-\eta\right) d \mu(t) \\
\quad+\int_{-\infty}^{\infty} n_{-}\left(\eta, A-A_{q_{0}}+t\left(B-B_{q_{0}}\right)\right) d \mu(t) \\
\leq \int_{-\infty}^{\infty} \operatorname{ind}\left(J-\eta+A_{q_{0}}+t B_{q_{0}}, J-\eta\right) d \mu(t) \\
\quad+n_{-}\left(\eta / 2, A-A_{q_{0}}\right)+\frac{2}{\pi \eta}\left\|B-B_{q_{0}}\right\|_{S_{1}} .
\end{aligned}
$$

By a calculation similar to (5.9), we get

$$
\sup _{\lambda \in \Delta_{1} \cup \Delta_{2}}\left\|A\left(2 q_{0} b+\lambda\right)-A_{q_{0}}\left(2 q_{0} b+\lambda\right)\right\|=O\left(b^{-1 / 2}\right),
$$

whence $n_{-}\left(\eta / 2, A-A_{q_{0}}\right)=0, \lambda \in \Delta_{1} \cup \Delta_{2}$, for all sufficiently large $b$. Next, by Lemma 8.2 (i),

$$
\begin{aligned}
& \sup _{\lambda \in \Delta_{1} \cup \Delta_{2}}\left\|B\left(2 q_{0} b+\lambda\right)-B_{q_{0}}\left(2 q_{0} b+\lambda\right)\right\|_{S_{1}} \\
& \quad=\sup _{\lambda \in \Delta_{1} \cup \Delta_{2}}\left\|\sum_{q=0}^{q_{0}-1} B_{q}\left(2 q_{0} b+\lambda\right)\right\|_{S_{1}}=O\left(b^{3 / 4}\right), \quad b \rightarrow \infty .
\end{aligned}
$$

This proves (8.1).

2. We will prove that, for each $\eta \in(0,1 / 2)$,

$$
\begin{aligned}
\limsup & \sup _{b \rightarrow \infty}\left\{b^{-1} \int_{-\infty}^{\infty} \operatorname{ind}\left(J-\eta+A_{q_{0}}+t B_{q_{0}}, J-\eta\right) d \mu(t)\right. \\
& \left.-\frac{1}{2 \pi} \int_{\mathbb{R}^{2}} d X_{\perp} \int_{-\infty}^{\infty} d \mu(t) \Xi_{\lambda}\left(2 \eta, X_{\perp}, t\right)\right\} \leq 0 .
\end{aligned}
$$

Fix $t \in \mathbb{R}$. Applying Corollary 7.2 Kreŭn's trace formula (1.1), and (3.8), we see that

$$
\begin{aligned}
& \sup _{\lambda \in \Delta_{1} \cup \Delta_{2}} \mid \int_{-\infty}^{\infty} b^{-1} \operatorname{ind}\left(J-\eta+A_{q_{0}}+t B_{q_{0}}, J-\eta\right) \phi(\eta) d \eta \\
&-\frac{1}{2 \pi} \int_{\mathbb{R}^{2}} d X_{\perp} \int_{-\infty}^{\infty} d \eta \Xi_{\lambda}\left(\eta, X_{\perp}, t\right) \phi(\eta) \mid \rightarrow 0
\end{aligned}
$$

for any polynomial $\phi$, as $b \rightarrow \infty$. Next, the bounds

$$
\sup _{b>0} \sup _{\lambda \in \Delta_{1} \cup \Delta_{2}}\left\|T_{q_{0}}\left(2 b q_{0}+\lambda\right)\right\|<\infty, \quad \sup _{X_{\perp} \in \mathbb{R}^{2}} \sup _{\lambda \in \Delta_{1} \cup \Delta_{2}}\left\|\tau\left(X_{\perp}, \lambda\right)\right\|<\infty
$$

ensure that for all $\lambda \in \Delta_{1} \cup \Delta_{2}$ the functions ind $\left(J-\eta+A_{q_{0}}+t B_{q_{0}}, J-\eta\right)$ and $\Xi_{\lambda}\left(\eta, X_{\perp}, t\right)$ vanish for all $\eta$ outside some fixed interval $(-R, R)$, where $R$ may depend on $q_{0}, V$, and $t$, but not on $b$ or $X_{\perp}$. Together with the fact that $\Xi_{\lambda}(\cdot, \cdot, t) \in L^{1}\left(\mathbb{R} \times \mathbb{R}^{2}, d \eta d X_{\perp}\right)$ (see 
Lemma 6.2(ii)), this allows us to change the order of integration in the second integral in (8.3). This yields

$$
\begin{array}{r}
\sup _{\lambda \in \Delta_{1} \cup \Delta_{2}} \mid \int_{-R}^{R} d \eta \phi(\eta)\left(b^{-1} \operatorname{ind}\left(J-\eta+A_{q_{0}}+t B_{q_{0}}, J-\eta\right)\right. \\
\left.-\frac{1}{2 \pi} \int_{\mathbb{R}^{2}} \Xi_{\lambda}\left(\eta, X_{\perp}, t\right) d X_{\perp}\right) \mid \rightarrow 0
\end{array}
$$

as $b \rightarrow \infty$.

3. We show that (8.4) extends to any $\phi \in C(-R, R)$. By (3.8), Krel̆n's inequality (see, e.g., 31, Theorem 8.2.1]), and Lemma 8.2(i), we obtain

$$
\begin{gathered}
\sup _{\lambda \in \Delta_{1} \cup \Delta_{2}} \int_{-R}^{R}\left|b^{-1} \operatorname{ind}\left(J-\eta+A_{q_{0}}+t B_{q_{0}}, J-\eta\right)\right| d \eta \\
\quad=\sup _{\lambda \in \Delta_{1} \cup \Delta_{2}} \int_{-R}^{R}\left|b^{-1} \xi\left(\eta ; J+A_{q_{0}}+t B_{q_{0}}, J\right)\right| d \eta \\
\quad \leq b^{-1} \sup _{\lambda \in \Delta_{1} \cup \Delta_{2}}\left\|A_{q_{0}}+t B_{q_{0}}\right\|_{S_{1}}=O(1)
\end{gathered}
$$

as $b \rightarrow \infty$. Thus, the norms of $b^{-1} \operatorname{ind}\left(J-\eta+A_{q_{0}}+t B_{q_{0}}, J-\eta\right)$ are uniformly bounded in $L^{1}(-R, R)$; so, by the Weierstrass theorem, we can extend (8.4) by continuity from the polynomials to all $\phi \in C(-R, R)$.

4. Consider 8.4 with an arbitrary $\phi \in C(-R, R)$ such that $\operatorname{supp} \phi \subset(-1 / 2,1 / 2)$. We apply the dominated convergence theorem to show that (8.4) can be integrated with respect to $d \mu(t)$. For any $\eta \in(-1 / 2,1 / 2)$, mimicking the pattern of (3.16) and (3.17), and applying Lemma 8.2 (ii), we show that

$$
\begin{gathered}
\left|b^{-1} \xi\left(\eta ; J+A_{q_{0}}+t B_{q_{0}}, J\right)\right| \leq b^{-1} n_{*}\left(1 / 2, A_{q_{0}}+t B_{q_{0}}\right) \\
\leq b^{-1}\left\|4 A_{q_{0}}\right\|_{S_{1}}+b^{-1}\left\|4 t B_{q_{0}}\right\|_{S_{p}}^{p} \leq C+C t^{p}
\end{gathered}
$$

uniformly over $\eta \in(-1 / 2,1 / 2)$ and $\lambda \in \Delta_{1} \cup \Delta_{2}$. Choosing $2 / m_{\perp}<p<1$, we get an integrable bound for the first term in (8.4). An integrable bound for the second term in (8.4) is provided by Lemma 6.2 Thus, (8.4) can be integrated with respect to $d \mu(t)$, which yields

$$
\begin{array}{r}
\sup _{\lambda \in \Delta_{1} \cup \Delta_{2}} \mid \int_{-\infty}^{\infty} d \mu(t) \int_{-1 / 2}^{1 / 2} d \eta \phi(\eta)\left(b^{-1} \operatorname{ind}\left(J-\eta+A_{q_{0}}+t B_{q_{0}}, J-\eta\right)\right. \\
\left.-\frac{1}{2 \pi} \int_{\mathbb{R}^{2}} \Xi_{\lambda}\left(\eta, X_{\perp}, t\right) d X_{\perp}\right) \mid \\
\leq \int_{-\infty}^{\infty} d \mu(t) \sup _{\lambda \in \Delta_{1} \cup \Delta_{2}} \mid \int_{-1 / 2}^{1 / 2} d \eta \phi(\eta)\left(b^{-1} \operatorname{ind}\left(J-\eta+A_{q_{0}}+t B_{q_{0}}, J-\eta\right)\right. \\
\left.-\frac{1}{2 \pi} \int_{\mathbb{R}^{2}} \Xi_{\lambda}\left(\eta, X_{\perp}, t\right) d X_{\perp}\right) \mid \rightarrow 0
\end{array}
$$

as $b \rightarrow \infty$. The bounds 8 (8.5) and (6.6) show that we can interchange the order of integration on the left in (8.6). Thus,

$$
\begin{array}{r}
\sup _{\lambda \in \Delta_{1} \cup \Delta_{2}} \mid \int_{-1 / 2}^{1 / 2} d \eta \phi(\eta) \int_{-\infty}^{\infty} d \mu(t)\left(\frac{1}{b} \operatorname{ind}\left(J-\eta+A_{q_{0}}+t B_{q_{0}}, J-\eta\right)\right. \\
\left.-\frac{1}{2 \pi} \int_{\mathbb{R}^{2}} \Xi_{\lambda}\left(\eta, X_{\perp}, t\right) d X_{\perp}\right) \mid \rightarrow 0
\end{array}
$$

as $b \rightarrow \infty$. 
5. By using the chain rule (3.7) and property (e) of ind, it is straightforward to see that the functions ind $\left(J-\eta+A_{q_{0}}+t B_{q_{0}}, J-\eta\right)$ and $\Xi_{\lambda}\left(\eta, X_{\perp}, t\right)$ are monotone nondecreasing in $\eta \in(-1,1)$. For a given $\eta_{0} \in(0,1 / 2)$, choose a continuous nonnegative function $\phi$ with support in $\left(\eta_{0}, 2 \eta_{0}\right)$ and such that $\int_{\eta_{0}}^{2 \eta_{0}} \phi(\eta) d \eta=1$. Then

$$
\begin{gathered}
\int_{-1 / 2}^{1 / 2} d \eta \phi(\eta) \int_{-\infty}^{\infty} d \mu(t)\left(b^{-1} \operatorname{ind}\left(J-\eta+A_{q_{0}}+t B_{q_{0}}, J-\eta\right)\right. \\
\left.-\frac{1}{2 \pi} \int_{\mathbb{R}^{2}} \Xi_{\lambda}\left(\eta, X_{\perp}, t\right) d X_{\perp}\right) \\
\geq \int_{-\infty}^{\infty} d \mu(t)\left(b^{-1} \operatorname{ind}\left(J-\eta_{0}+A_{q_{0}}+t B_{q_{0}}, J-\eta_{0}\right)-\frac{1}{2 \pi} \int_{\mathbb{R}^{2}} \Xi_{\lambda}\left(2 \eta_{0}, X_{\perp}, t\right) d X_{\perp}\right) .
\end{gathered}
$$

Combining this with 8.7), we get 8.2).

6. Combining (8.1), (8.2), and (3.21), we obtain

$$
\begin{aligned}
\limsup _{b \rightarrow \infty} \operatorname{ess}_{\sup } \operatorname{su\Delta }_{\lambda \in \Delta_{1} \cup \Delta_{2}}\{ & b^{-1} \xi\left(2 b q_{0}+\lambda ; H(b), H_{0}(b)\right) \\
& \left.-\frac{1}{2 \pi} \int_{\mathbb{R}^{2}} d X_{\perp} \int_{-\infty}^{\infty} d \mu(t) \Xi_{\lambda}\left(2 \eta, X_{\perp}, t\right)\right\} \leq 0 .
\end{aligned}
$$

Similarly,

$$
\begin{aligned}
\liminf _{b \rightarrow \infty} \operatorname{essinf} \inf _{\lambda \in \Delta_{1} \cup \Delta_{2}}\{ & b^{-1} \xi\left(2 b q_{0}+\lambda ; H(b), H_{0}(b)\right) \\
& \left.-\frac{1}{2 \pi} \int_{\mathbb{R}^{2}} d X_{\perp} \int_{-\infty}^{\infty} d \mu(t) \Xi_{\lambda}\left(-2 \eta, X_{\perp}, t\right)\right\} \geq 0 .
\end{aligned}
$$

The subsequent arguments are different for $\Delta_{1}$ and $\Delta_{2}$. First, we consider the interval $\Delta_{1}$. By Lemma [6.3)(ii), the integral $\int_{\mathbb{R}^{2}} d X_{\perp} \int_{-\infty}^{\infty} d \mu(t) \Xi_{\lambda}\left(\eta, X_{\perp}, t\right)$ is continuous in $(\lambda, \eta) \in \Delta_{1} \times[-1 / 2,1 / 2]$ and therefore is uniformly continuous on this set. It follows that

$$
\sup _{\lambda \in \Delta_{1} \cup \Delta_{2}}\left|\int_{\mathbb{R}^{2}} d X_{\perp} \int_{-\infty}^{\infty} d \mu(t)\left(\Xi_{\lambda}\left(\eta, X_{\perp}, t\right)-\Xi_{\lambda}\left(0, X_{\perp}, t\right)\right)\right| \rightarrow 0 \text { as } \eta \rightarrow 0 .
$$

Combining (8.8), (8.9), recalling that $\eta$ can be taken arbitrarily small, and using (8.10) and (6.4), we obtain the desired limit relation 2.2).

7. Finally, we employ (8.8) and (8.9) to prove relations (2.3) and (2.4). Note that here $\lambda<0$, so that $\beta\left(X_{\perp}, \lambda\right)=0$. For each $\eta \in(-1,1)$ and $X_{\perp} \in \mathbb{R}^{2}$, using the representation (3.15), formula (1.3), and a continuity argument, we obtain

$$
\begin{aligned}
\Xi_{\lambda}\left(\eta, X_{\perp}, t\right) & =\operatorname{ind}\left(\iota\left(X_{\perp}\right)+\alpha\left(X_{\perp}, \lambda\right)-\eta, \iota\left(X_{\perp}\right)-\eta\right) \\
& =-\lim _{\epsilon \rightarrow+0} N\left(\lambda+\epsilon ; \chi_{0}+\left|V\left(X_{\perp}, \cdot\right)\right|\left(\iota\left(X_{\perp}\right)-\eta\right)^{-1}\right) .
\end{aligned}
$$

Next, for $|\eta| \leq 1 / 2$ we have

$$
\begin{aligned}
N(\lambda & \left.+\epsilon ; \chi_{0}+\left|V\left(X_{\perp}, \cdot\right)\right|\left(\iota\left(X_{\perp}\right)-\eta\right)^{-1}\right) \\
& =N\left(\lambda+\epsilon ; \chi_{0}+V\left(X_{\perp}, \cdot\right)+\left|V\left(X_{\perp}, \cdot\right)\right| \eta\left(1-\iota\left(X_{\perp}\right) \eta\right)^{-1}\right) \\
& \geq N\left(\lambda+\epsilon-2 C_{0}|\eta|, \chi_{0}+V\left(X_{\perp}, \cdot\right)\right) .
\end{aligned}
$$

Therefore,

$$
\Xi_{\lambda}\left(\eta, X_{\perp}, t\right) \leq \Xi_{\lambda-2 C_{0}|\eta|}\left(0, X_{\perp}, t\right) .
$$

Similarly, for $|\eta| \leq 1 / 2,2 C_{0} \eta<-\lambda$, we obtain

$$
\Xi_{\lambda}\left(\eta, X_{\perp}, t\right) \geq \Xi_{\lambda+2 C_{0}|\eta|}\left(0, X_{\perp}, t\right) .
$$

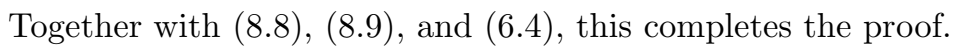




\section{§9. Spectral properties of the Landau Hamiltonian}

9.1. Creation and annihilation operators. Angular momentum eigenbases. In this subsection we construct orthonormal bases of the subspaces $\mathcal{H}_{q}:=\operatorname{Ker}(h(g)-2 b q)=$ $p_{q} L^{2}\left(\mathbb{R}^{2}\right), q \in \mathbb{Z}_{+}$(see Subsection 4.1), known in the physics literature as the angularmomentum eigenbases (see [13, 17]). For $\mathbf{x}=\left(x_{1}, x_{2}\right) \in \mathbb{R}^{2}$, we take the complex variable $\zeta:=x_{1}+i x_{2}$, set

$$
\partial:=\frac{\partial}{\partial \zeta}=\frac{1}{2}\left(\frac{\partial}{\partial x_{1}}-i \frac{\partial}{\partial x_{2}}\right), \quad \bar{\partial}:=\frac{\partial}{\partial \bar{\zeta}}=\frac{1}{2}\left(\frac{\partial}{\partial x_{1}}+i \frac{\partial}{\partial x_{2}}\right),
$$

and introduce the annihilation and the creation operators

$$
\begin{gathered}
a=a(b):=-2 i\left(\bar{\partial}+\frac{b}{4} \zeta\right)=-2 i e^{-b|\zeta|^{2} / 4} \bar{\partial} e^{b|\zeta|^{2} / 4}, \\
a^{*}=a(b)^{*}:=-2 i\left(\partial-\frac{b}{4} \bar{\zeta}\right)=-2 i e^{b|\zeta|^{2} / 4} \partial e^{-b|\zeta|^{2} / 4},
\end{gathered}
$$

defined originally on the Schwartz class $\mathcal{S}\left(\mathbb{R}^{2}\right)$ and then closed in $L^{2}\left(\mathbb{R}^{2}\right)$. It is easy to check that the operators $a$ and $a^{*}$ are mutually adjoint. Moreover, on $\mathcal{S}\left(\mathbb{R}^{2}\right)$ we have

$$
\left[a(b), a(b)^{*}\right]=2 b,
$$

and $h(b)=a^{*} a$. Therefore, the common domain of the closed operators $a$ and $a^{*}$ coincides with the domain of $h(b)^{1 / 2}$. A standard argument from the representation theory of the Heisenberg algebra yields $\mathcal{H}_{0}=\operatorname{Ker} a, \mathcal{H}_{q}=\left(a^{*}\right)^{q} \mathcal{H}_{0}, q \geq 1$ (see, e.g., 6, 5.2]). Moreover, Ker $a=\left\{f \in L^{2}\left(\mathbb{R}^{2}\right) \mid f=g e^{-b|\mathbf{x}|^{2} / 4}, \bar{\partial} g=0\right\}$. Therefore, it is easily seen that the functions

$$
\varphi_{0, k}(\mathbf{x}):=\frac{1}{\sqrt{\pi k !}}\left(\frac{b}{2}\right)^{(k+1) / 2}\left(x_{1}+i x_{2}\right)^{k} e^{-b|\mathbf{x}|^{2} / 4}, \quad \mathbf{x} \in \mathbb{R}^{2}, \quad k \in \mathbb{Z}_{+},
$$

form an orthonormal basis of $\mathcal{H}_{0}$, while the functions

$$
\varphi_{q, k}(\mathbf{x}):=\frac{1}{\sqrt{(2 b)^{q} q !}}\left(\left(a^{*}\right)^{q} \varphi_{0, k}\right)(\mathbf{x}), \quad \mathbf{x} \in \mathbb{R}^{2}, \quad k \in \mathbb{Z}_{+}
$$

form an orthonormal basis of $\mathcal{H}_{q}, q \geq 1$. The functions $\varphi_{q, k}$ can be written in the polar coordinates $(\rho, \theta)$ :

$$
\varphi_{q, k}(\rho \cos \theta, \rho \sin \theta)=(-i)^{q} \sqrt{\frac{q !}{\pi k !}\left(\frac{b}{2}\right)^{k-q+1}} e^{i(k-q) \theta} \rho^{k-q} L_{q}^{(k-q)}\left(b \rho^{2} / 2\right) e^{-b \rho^{2} / 4}
$$

where

$$
L_{q}^{(k-q)}(\xi):=\sum_{l=\max \{0, q-k\}}^{q} \frac{k !}{(k-q+l) !(q-l) !} \frac{(-\xi)^{l}}{l !}, \quad \xi \geq 0, \quad q \in \mathbb{Z}_{+}, \quad k \in \mathbb{Z}_{+},
$$

are the generalized Laguerre polynomials.

9.2. Proof of Lemma [5.2. Lemma 5.2 is an immediate consequence of the following fact.

Lemma 9.1. Let $v \in \operatorname{Lip}\left(\mathbb{R}^{2}\right)$ be such that $\sup _{x \in \mathbb{R}^{2}}(|v(x)|+|\nabla v(x)|)<\infty$. Fix $q \in \mathbb{Z}_{+}$. Then

$$
\left\|\left(1-p_{q}\right) v p_{q}\right\| \leq b^{-1 / 2} \sqrt{2}(\sqrt{q+1}+\sqrt{q}) \sup _{\mathbf{x} \in \mathbb{R}^{2}}|\nabla v(\mathbf{x})|
$$


Proof. Without loss of generality, we may assume that $v \in C^{\infty}\left(\mathbb{R}^{2}\right)$ and that all its derivatives are uniformly bounded on $\mathbb{R}^{2}$. We have $\left(1-p_{q}\right) v p_{q}=\left(1-p_{q}\right)\left[v, p_{q}\right] p_{q}$, where $\left[v, p_{q}\right]:=v p_{q}-p_{q} v$ denotes the commutator of the operators $v$ and $p_{q}$. Suppose that $2 b>1$. Then $p_{q}=\frac{1}{2 \pi i} \int_{\Gamma_{q}}(z-h)^{-1} d z$, where $\Gamma_{q}$ is the circle $\{z \in \mathbb{C}|| z-2 b q \mid=1\}$ run over in the positive direction. Therefore,

$$
\begin{aligned}
{\left[v, p_{q}\right] } & =-\frac{1}{2 \pi i} \int_{\Gamma_{q}}\left[(z-h)^{-1}, v\right] d z \\
& =-\frac{1}{2 \pi i} \int_{\Gamma_{0}}(z+2 b q-h)^{-1}[h, v](z+2 b q-h)^{-1} d z .
\end{aligned}
$$

Since $h=a^{*} a$, we get $[h, v]=\left[a^{*} a, v\right]=a^{*}[a, v]+\left[a^{*}, v\right] a=-2 i\left(a^{*} \bar{\partial} V+\partial V a\right)$. Hence,

$$
\left(1-p_{q}\right)\left[v, p_{q}\right] p_{q}=2 i(h-2 b q)^{-1}\left(1-p_{q}\right) a^{*} \bar{\partial} V p_{q}+2 i(h-2 b q)^{-1}\left(1-p_{q}\right) \partial V a p_{q} .
$$

It is easy to check that

$$
\begin{gathered}
\left\|(h-2 b q)^{-1}\left(1-p_{q}\right) a^{*}\right\|=\left\|a(h-2 b q)^{-1}\left(1-p_{q}\right)\right\|=\sqrt{\frac{q+1}{2 b}}, \quad\left\|p_{q}\right\|=1, \\
\left\|(h-2 b q)^{-1}\left(1-p_{q}\right)\right\|=\frac{1}{2 b}, \quad\left\|a p_{q}\right\|=\sqrt{2 b q} .
\end{gathered}
$$

Combining (9.9) with (9.10)-9.11), we immediately get (9.8).

9.3. Unitary equivalence of Toeplitz operators corresponding to different Landau levels. By $\langle f, g\rangle:=\int_{\mathbb{R}^{2}} f \bar{g} d \mathbf{x}, f, g \in L^{2}\left(\mathbb{R}^{2}\right)$, we denote the scalar product in $L^{2}\left(\mathbb{R}^{2}\right)$. Moreover, we use the notation $(f, g):=\langle f, \bar{g}\rangle, f, g \in L^{2}\left(\mathbb{R}^{2}\right)$. If $g \in \mathcal{S}\left(\mathbb{R}^{2}\right)$ is fixed, then the functionals $(f, g)$ and $\langle f, g\rangle$ can be extended by continuity to $f \in \mathcal{S}^{\prime}\left(\mathbb{R}^{2}\right)$. Note also that if $f \in \mathcal{S}^{\prime}\left(\mathbb{R}^{2}\right), h \in \mathcal{S}\left(\mathbb{R}^{2}\right)$, then the product $f h$ is a well-defined element of $\mathcal{S}^{\prime}\left(\mathbb{R}^{2}\right)$, acting in accordance with $(f h, g)=(f, h g)$.

Lemma 9.2. Let $F \in \mathcal{S}^{\prime}\left(\mathbb{R}^{2}\right)$, and let $j, k, q \in \mathbb{Z}_{+}$. Then

$$
\left\langle F \varphi_{q, k}, \varphi_{q, j}\right\rangle=\left\langle\left(\mathcal{D}_{q} F\right) \varphi_{0, k}, \varphi_{0, j}\right\rangle,
$$

where

$$
\left(\mathcal{D}_{q} F\right)(\mathbf{x})=\sum_{s=0}^{q} d_{s, q}\left(\Delta^{s} F\right)(\mathbf{x}), \quad \mathbf{x} \in \mathbb{R}^{2},
$$

and

$$
d_{s, q}:=\frac{q !}{(s !)^{2}(q-s) !}(2 b)^{-s} .
$$

Proof. First, note that the functions $\varphi_{q, k} \bar{\varphi}_{q, j}$ as well as $\varphi_{0, k} \bar{\varphi}_{0, j}$ are in the Schwartz class $\mathcal{S}\left(\mathbb{R}^{2}\right)$. Therefore, we may assume without loss of generality that $F \in \mathcal{S}\left(\mathbb{R}^{2}\right)$, because $\mathcal{S}\left(\mathbb{R}^{2}\right)$ is dense in $\mathcal{S}^{\prime}\left(\mathbb{R}^{2}\right)$, and $\mathcal{D}_{q}: \mathcal{S}^{\prime}\left(\mathbb{R}^{2}\right) \rightarrow \mathcal{S}^{\prime}\left(\mathbb{R}^{2}\right)$ is a continuous mapping. Then all the functions $\left(D^{\alpha} F\right) \varphi_{s, k} \equiv \frac{\left(D^{\alpha} F\right)\left(a^{*}\right)^{s} \varphi_{0, k}}{\sqrt{s !(2 b)^{s}}}$ with $\alpha \in \mathbb{Z}_{+}^{2}, s \in \mathbb{Z}_{+}, k \in \mathbb{Z}_{+}$are in $\mathcal{S}\left(\mathbb{R}^{2}\right)$ and therefore in the domain of the operators $a$ and $a^{*}$. Next, relations (9.1)-(9.2) imply

$$
[a, F]=-[F, a]=-2 i \bar{\partial} F, \quad\left[a^{*}, F\right]=-\left[F, a^{*}\right]=-2 i \partial F,
$$

whence

$$
\left[[a, F], a^{*}\right]=-2 i\left[\bar{\partial} F, a^{*}\right]=4 \partial \bar{\partial} F=\Delta F .
$$

Fix $j, k, q \in \mathbb{Z}_{+}$. First, we use induction on $q \in \mathbb{Z}_{+}$to prove that (9.12) is valid with coefficients $d_{s, q}$ depending only on $s, q$, and $b$. 
Fixing $Q \in \mathbb{Z}_{+}$, we assume that $(9.12)$ is valid with coefficients $d_{s, q}$ that depend only on $s, q$, and $b$ for every $q=0, \ldots, Q$. We shall prove that similar relations hold true for $q=Q+1$. We have

$$
\left\langle F \varphi_{Q+1, k}, \varphi_{Q+1, j}\right\rangle=\frac{1}{(Q+1) !(2 b)^{Q+1}}\left\langle F\left(a^{*}\right)^{Q+1} \varphi_{0, k},\left(a^{*}\right)^{Q+1} \varphi_{0, j}\right\rangle .
$$

Utilizing the commutation relations (9.3), (9.16), and (9.15), and taking into account the fact that $\varphi_{0, j}$ is in $\operatorname{Ker} a$, we see that

$$
\begin{aligned}
\left\langle F\left(a^{*}\right)^{Q+1} \varphi_{0, k},\left(a^{*}\right)^{Q+1} \varphi_{0, j}\right\rangle & \\
= & 2 b(Q+1)\left\langle F\left(a^{*}\right)^{Q} \varphi_{0, k},\left(a^{*}\right)^{Q} \varphi_{0, j}\right\rangle+\left\langle[a, F]\left(a^{*}\right)^{Q+1} \varphi_{0, j},\left(a^{*}\right)^{Q} \varphi_{0, k}\right\rangle \\
= & 2 b(Q+1)\left\langle F\left(a^{*}\right)^{Q} \varphi_{0, k},\left(a^{*}\right)^{Q} \varphi_{0, j}\right\rangle \\
& +\left\langle\Delta F\left(a^{*}\right)^{Q} \varphi_{0, k},\left(a^{*}\right)^{Q} \varphi_{0, j}\right\rangle+2 b Q\left\langle[a, F]\left(a^{*}\right)^{Q} \varphi_{0, k},\left(a^{*}\right)^{Q-1} \varphi_{0, j}\right\rangle
\end{aligned}
$$

if $Q>0$; if $Q=0$, the last term should be set equal to zero. By recurrence,

$$
\begin{aligned}
& \left\langle F\left(a^{*}\right)^{Q+1} \varphi_{0, k},\left(a^{*}\right)^{Q+1} \varphi_{0, j}\right\rangle \\
& =2 b(Q+1)\left\langle F\left(a^{*}\right)^{Q} \varphi_{0, k},\left(a^{*}\right)^{Q} \varphi_{0, j}\right\rangle \\
& \quad+Q !(2 b)^{Q} \sum_{q=0}^{Q} \frac{1}{(2 b)^{q} q !}\left\langle\Delta F\left(a^{*}\right)^{q} \varphi_{0, k},\left(a^{*}\right)^{q} \varphi_{0, j}\right\rangle .
\end{aligned}
$$

Combining (9.17) with (9.18), we get

$$
\begin{aligned}
\left\langle F \varphi_{Q+1, k}, \varphi_{Q+1, j}\right\rangle & =\left\langle F \varphi_{Q, k}, \varphi_{Q, j}\right\rangle+\frac{1}{2 b(Q+1)} \sum_{q=0}^{Q}\left\langle\Delta F \varphi_{q, k}, \varphi_{q, j}\right\rangle \\
& =\sum_{s=0}^{Q+1} d_{s, Q+1}\left\langle\Delta^{s} F \varphi_{0, k}, \varphi_{0, j}\right\rangle
\end{aligned}
$$

with

$$
d_{s, Q+1}:= \begin{cases}d_{0, Q} & \text { if } s=0, \\ \frac{1}{2 b(Q+1)} \sum_{n=s}^{Q+1} d_{s-1, n-1}+d_{s, Q} & \text { if } 1 \leq s \leq Q, \\ \frac{1}{2 b(Q+1)} d_{Q, Q} & \text { if } s=Q+1 .\end{cases}
$$

Evidently, $d_{s, Q+1}$ depends only on $s, Q$, and $b$. In order to demonstrate that the numerical values of the coefficients $d_{s, q}$ are given by (9.13), we proceed in the following way. Set $F_{p}(\mathbf{x}):=|\mathbf{x}|^{2 p}, p=0, \ldots, q$. Then the identities

$$
\left\langle F_{p} \varphi_{q, 0}, \varphi_{q, 0}\right\rangle=\left\langle\left(\mathcal{D}_{q} F_{p}\right) \varphi_{0,0}, \varphi_{0,0}\right\rangle=\sum_{s=0}^{q} d_{s, q}\left\langle\left(\Delta^{s} F_{p}\right) \varphi_{0,0}, \varphi_{0,0}\right\rangle, \quad p=0, \ldots, q,
$$

are special cases of (19.12). A straightforward calculation yields

$$
\begin{aligned}
\left\langle F_{p} \varphi_{q, 0}, \varphi_{q, 0}\right\rangle & =\frac{(p+q) !}{q !}(b / 2)^{-p}, \\
\left\langle\left(\Delta^{s} F_{p}\right) \varphi_{0,0}, \varphi_{0,0}\right\rangle & = \begin{cases}4^{s} \frac{(p !)^{2}}{(p-s) !}(b / 2)^{s-p} & \text { if } 0 \leq s \leq p, \\
0 & \text { if } p<s \leq q .\end{cases}
\end{aligned}
$$

Therefore, from (9.19) it follows that the $d_{s, q}, s=0, \ldots, q$, yield a unique solution of the linear system

$$
\sum_{s=0}^{p} \frac{4^{s}(p !)^{2}}{(p-s) !}(b / 2)^{s-p} d_{s, q}=\frac{(p+q) !}{q !}(b / 2)^{-p}, \quad p=0, \ldots, q .
$$


Setting

$$
d_{s, q}=(2 b)^{-s} h_{s, q}, \quad s=0, \ldots, q,
$$

we find that (9.20) is equivalent to the system

$$
\sum_{s=0}^{p} \frac{p !}{(p-s) !} h_{s, q}=\left(\begin{array}{c}
p+q \\
p
\end{array}\right), \quad p=0, \ldots, q .
$$

Invoking the elementary combinatorial identity $\sum_{s=0}^{p}\left(\begin{array}{c}q \\ s\end{array}\right)\left(\begin{array}{c}p \\ p-s\end{array}\right)=\left(\begin{array}{c}p+q \\ p\end{array}\right)$ (see, e.g., [16 equation 0.165$]$ ), we conclude that the solution of $(9.22)$ is given by

$$
h_{s, q}=\left(\begin{array}{l}
q \\
s
\end{array}\right) \frac{1}{s !}, \quad s=0, \ldots, q .
$$

Combination of (9.21) and (9.23) yields (9.14).

Corollary 9.3. Let $q \in \mathbb{Z}_{+}$, and let $F$ be the operator of multiplication by a real function $F \in C^{2 q}\left(\mathbb{R}^{2}\right)$. Assume that $\Delta^{s} F \in L^{\infty}\left(\mathbb{R}^{2}\right), s=0, \ldots, q$. Then the operator $p_{q}(b) F p_{q}(b): L^{2}\left(\mathbb{R}^{2}\right) \rightarrow L^{2}\left(\mathbb{R}^{2}\right)$ is unitarily equivalent to the operator $p_{0}(b)\left(\mathcal{D}_{q} F\right) p_{0}(b):$ $L^{2}\left(\mathbb{R}^{2}\right) \rightarrow L^{2}\left(\mathbb{R}^{2}\right)$, where $\mathcal{D}_{q}$ is the differential operation defined in (9.13)-(9.14).

9.4. Proof of Lemma 8.1 For $\theta>0$ and $q \in \mathbb{Z}_{+}$, we define an operator $\mathcal{G}_{q, \theta, b}$ : $L^{2}\left(\mathbb{R}^{2}\right) \rightarrow L^{2}\left(\mathbb{R}^{2}\right)$ by

$$
\mathcal{G}_{q, \theta, b}:=p_{q}(b) W_{\theta} p_{q}(b),
$$

where $W_{\theta}$ is the operator of multiplication by the function $\langle\mathbf{x}\rangle^{-2 / \theta}, \mathbf{x} \in \mathbb{R}^{2}$. It is easy to check that $\mathcal{G}_{q, \theta, b}$ is compact, selfadjoint, and nonnegative (see [24, Lemma 5.1]).

Now, Lemma 8.1 is a direct consequence of the following fact.

Lemma 9.4. Suppose $\theta>0, q \in \mathbb{Z}_{+}, s>0$, and $b \geq 1 / 2$. Then

$$
n_{+}\left(s ; \mathcal{G}_{q, \theta, b}\right) \leq C_{3}+C_{4} b s^{-\theta},
$$

where $C_{3}=C_{3}(\theta)$ and $C_{4}=C_{4}(q, \theta)$ are independent of $s$ and $b$.

Proof. By Lemma 9.3 the nonzero eigenvalues of the operator $\mathcal{G}_{q, \theta, b}$ coincide with the nonzero eigenvalues of the operator $\tilde{\mathcal{G}}_{q, \theta, b}=p_{0}(b)\left(\mathcal{D}_{q} W_{\theta}\right) p_{0}(b)$. Evidently, there exists a function $w_{q, \theta, b}:[0, \infty) \rightarrow \mathbb{R}$ different from zero almost everywhere and such that $\left(\mathcal{D}_{q} W_{\theta}\right)(\mathbf{x})=w_{q, \theta, b}\left(|\mathbf{x}|^{2}\right), \mathbf{x} \in \mathbb{R}^{2}$. By [28, Lemma 3.3], the nonzero eigenvalues $\nu_{k}$ of the operator $\tilde{\mathcal{G}}_{q, \theta, b}$ can be written as

$$
\nu_{k}=\nu_{k}(q, \theta, b):=\frac{1}{k !} \int_{0}^{\infty} w_{q, b, \theta}(2 \eta / b) e^{-\eta} \eta^{k} d \eta, \quad k \in \mathbb{Z}_{+} .
$$

Since $b / 2 \geq 1$, we have $d_{s, q} \leq q$ ! (see (9.13)-(9.14) ). Hence, $w_{q, b, \theta}(t) \leq C_{5}\langle t\rangle^{-1 / \theta}, t \in \mathbb{R}$, with a constant $C_{5}$ that may depend on $q$ and $\theta$ but not on $b$ and $t$. Therefore, (9.25) implies

$$
\begin{array}{r}
\nu_{k}(q, b, \theta)=\frac{1}{k !} \int_{0}^{\infty} w_{q, b, \theta}(2 \eta / b) e^{-\eta} \eta^{k} d \eta \leq \frac{C_{5}}{k !} \int_{0}^{\infty}\langle 2 \eta / b\rangle^{-1 / \theta} e^{-\eta} \eta^{k} d \eta \\
\leq C_{5}\left(\frac{b}{2}\right)^{1 / \theta} \frac{1}{k !} \int_{0}^{\infty} e^{-\eta} \eta^{k-1 / \theta} d \eta=\frac{C_{5}}{2^{1 / \theta}} b^{1 / \theta} \frac{\Gamma(k+1-1 / \theta)}{\Gamma(k+1)}, \\
k \in \mathbb{Z}_{+}, \quad k>-1+1 / \theta .
\end{array}
$$


Since $\lim _{k \rightarrow \infty} k^{1 / \theta} \frac{\Gamma(k+1-1 / \theta)}{\Gamma(k+1)}=1$ (see [1] equation (6.1.46)]), there exists $k_{0}=k_{0}(\theta) \geq 1$ such that for $k \geq k_{0}$ we have $\nu_{k}(q, \theta, b) \leq \frac{2 C_{5}}{2^{1 / \theta}}(k / b)^{-1 / \theta}$. Therefore,

$$
\begin{aligned}
& n_{+}\left(s ; \mathcal{G}_{q, b, \theta}\right)=n_{+}\left(s ; \tilde{\mathcal{G}}_{q, b, \theta}\right)=\#\left\{k \in \mathbb{Z}_{+} \mid \nu_{k}(q, \theta, b)>s\right\} \\
& \quad \leq k_{0}+\#\left\{k>k_{0} \mid \frac{2 C_{5}}{2^{1 / \theta}}(k / b)^{-1 / \theta}>s\right\} \leq k_{0}+\frac{1}{2}\left(2 C_{5}\right)^{\theta} b s^{-\theta},
\end{aligned}
$$

which is equivalent to (9.24) with $C_{3}=k_{0}, C_{4}=\left(2 C_{5}\right)^{\theta} / 2$.

\section{REFERENCES}

[1] M. Abramowitz and I. Stegun (eds.), Handbook of mathematical functions with formulas, graphs, and mathematical tables, Nat. Bureau of Standards Appl. Math. Ser., vol. 55, U. S. Government Printing Office, Washington, DC, 1964. MR0167642 (29:4914)

[2] S. Agmon, Spectral properties of Schrödinger operators and scattering theory, Ann. Scuola Norm. Sup. Pisa Cl. Sci. (4) 2 (1975), no. 2, 151-218. MR0397194 (53:1053)

[3] J. Avron, I. Herbst, and B. Simon, Schrödinger operators with magnetic fields. I. General interactions, Duke Math. J. 45 (1978), 847-883. MR0518109 (80k:35054)

[4] J. Avron, R. Seiler, and B. Simon, The index of a pair of projections, J. Funct. Anal. 120 (1994), no. 1, 220-237. MR1262254 (95b:47012)

[5] A. Balazard-Konlein, Calcul fonctionnel pour des opérateurs $h$-admissibles à symbole opérateur et applications, Thèse de Docteur de 3ème cycle, Univ. de Nantes, 1985.

[6] F. A. Berezin and M. A. Shubin, The Schrödinger equation, Moskov. Gos. Univ., Moscow, 1983; English transl., Math. Appl. (Soviet Ser.), vol. 66, Kluwer Acad. Publishers Group, Dordrecht, 1991. MR.1186643 (93i:81001)

[7] M. Sh. Birman, On the spectrum of singular boundary value problems, Mat. Sb. 55 (1961), no. 2, 125-174; English transl., Amer. Math. Soc. Transl. (2), vol. 53, Amer. Math. Soc., Providence, RI, 1966, pp. 23-80. MR0142896 (26:463)

[8] M. Sh. Birman and S. B. Entina, Stationary approach in abstract scattering theory, Izv. Akad. Nauk SSSR Ser. Mat. 31 (1967), no. 2, 401-430; English transl., Math. USSR-Izv. 1 (1967), no. 1, 391-420. MR0209895|(35:790

[9] M. Sh. Birman and M. G. Krein, On the theory of wave operators and scattering operators, Dokl. Akad. Nauk SSSR 144 (1962), no. 3, 475-478; English transl., Soviet Math. Dokl. 3 (1962), 740-744. MR 0139007 (25:2447)

[10] M. Sh. Birman and D. R. Yafaev, The spectral shift function. The work of M. G. Krein and its further development, Algebra i Analiz 4 (1992), no. 5, 1-44; English transl., St. Petersburg Math. J. 4 (1993), no. 5, 833-870. MF 1202723 (94g:47002)

[11] V. S. Buslaev and L. D. Faddeev, Formulas for traces for a singular Sturm-Liouville differential operator, Dokl. Akad. Nauk SSSR 132 (1960), no. 1, 13-16; English transl., Soviet Math. Dokl. 1 (1960), 451-454. MR0120417 (22:11171)

[12] M. Dimassi and J. Sjöstrand, Spectral asymptotics in the semi-classical limit, London Math. Soc. Lecture Note Ser., vol. 268, Cambridge Univ. Press, Cambridge, 1999. MR1735654 (2001b:35237)

[13] V. Fock, Bemerkung zur Quantelung des harmonischen Oszillators im Magnetfeld, Z. Physik 47 (1928), 446-448.

[14] C. Gérard and I. Łaba, Multiparticle quantum scattering in constant magnetic fields, Math. Surveys Monographs, vol. 90, Amer. Math. Soc., Providence, RI, 2002. MR1871447 (2003d:81316)

[15] F. Gesztesy and K. Makarov, The $\Xi$ operator and its relation to Krein's spectral shift function, J. Anal. Math. 81 (2000), 139-183. MR.1785280 (2001i:47016)

[16] I. S. Gradshtein and I. M. Ryzhik, Tables of integrals, sums, series, and products, Fizmatgiz, Moscow, 1963; English transl., Acad. Press, New York-London, 1965. MR0161996 (28:5198) MR.0197789(33:5952)

[17] T. Hupfer, H. Leschke, and S. Warzel, Upper bounds on the density of states of single Landau levels broadened by Gaussian random potentials, J. Math. Phys. 42 (2001), 5626-5641. MR1866676 (2003b:82033)

[18] V. Ivrii, Microlocal analysis and precise spectral asymptotics, Springer Monographs in Math., Springer-Verlag, Berlin, 1998. MR,1631419 (99e:58193)

[19] M. G. Krel̆n, On the trace formula in perturbation theory, Mat. Sb. 33 (75) (1953), no. 3, 597-626. (Russian) MF0060742(15:720b)

[20] L. Landau, Diamagnetismus der Metalle, Z. Physik 64 (1930), 629-637. 
[21] A. B. Pushnitskii, Representation of the spectral shift function for perturbations of fixed sign, Algebra i Analiz 9 (1997), no. 6, 197-213; English transl., St. Petersburg Math. J. 9 (1998), no. 6, 1181-1194. MR 1722328 (2000j:47086)

[22] _ Estimates for the spectral shift function of the polyharmonic operator, J. Math. Phys. 40 (1999), 5578-5592. MR 1722328 (2000j:47086)

[23] _ The spectral shift function and the invariance principle, J. Funct. Anal. 183 (2001), 269320. MR.1844210 (2002j:47019)

[24] G. D. Raikov, Eigenvalue asymptotics for the Schrödinger operator with homogeneous magnetic potential and decreasing electric potential. I. Behaviour near the essential spectrum tips, Comm. Partial Differential Equations 15 (1990), 407-434; Errata: Comm. Partial Differential Equations 18 (1993), 1977-1979. MR 1044429 (91k:35188)

[25] _ Eigenvalue asymptotics for the Schrödinger operator in strong constant magnetic fields, Comm. Partial Differential Equations 23 (1998), no. 9-10, 1583-1619. MR1641780 (99i:35120)

[26] _ Asymptotic properties of the magnetic integrated density of states, Electron. J. Differential Equations 1999, No. 13, 27 pp. MR1679559|(2000h:35126)

[27] G. D. Raikov and M. Dimassi, Spectral asymptotics for quantum Hamiltonians in strong magnetic fields, Cubo Mat. Educ. 3 (2001), 317-391. MR1961594 (2004a:81092)

[28] G. D. Raikov and S. Warzel, Quasi-classical versus non-classical spectral asymptotics for magnetic Schrödinger operators with decreasing electric potentials, Rev. Math. Phys. 14 (2002), 1051-1072. MR.1939760 (2003m:81069)

[29] M. Reed and B. Simon, Methods of modern mathematical physics. IV. Analysis of operators, Acad. Press, New York-London, 1978. MR0493421 (58:12429c)

[30] A. V. Sobolev, Efficient bounds for the spectral shift function, Ann. Inst. H. Poincaré Phys. Théor. 58 (1993), no. 1, 55-83. MR 1208792 (94c:47018)

[31] D. R. Yafaev, Mathematical scattering theory. General theory, Transl. Math. Monogr., vol. 105, Amer. Math. Soc., Providence, RI, 1992. MR1180965 (94f:47012)

Mathématiques Appliquées de Bordeaux, Université Bordeaux I, 351 Cours de la libération, 33405 Talence, France

E-mail address: vbruneau@math.u-bordeaux.fr

Department of Mathematical Sciences, Loughborough University, Loughborough, LeicesTERShiRe, LE11 3TU, United Kingdom

E-mail address: A.B.Pushnitski@lboro.ac.uk

Departamento de Matemáticas, Universidad de Chile, Las Palmeras 3425, Casilla 653, SanTIAGO, Chile

E-mail address: graykov@uchile.cl

Received 27/OCT/2003

Originally published in English 